\title{
FIXED-PARAMETER TRACTABILITY AND COMPLETENESS I: BASIC RESULTS*
}

\author{
ROD G. DOWNEY ${ }^{\dagger}$ AND MICHAEL R. FELLOWS ${ }^{\ddagger}$
}

\begin{abstract}
For many fixed-parameter problems that are trivially soluable in polynomial time, such as $(k$-)DOMINATING SET, essentially no better algorithm is presently known than the one which tries all possible solutions. Other problems, such as $(k$-)FEEDBACK VERTEX SET, exhibit fixed-parameter tractability: for each fixed $k$ the problem is soluable in time bounded by a polynomial of degree $c$, where $c$ is a constant independent of $k$. We establish the main results of a completeness program which addresses the apparent fixed-parameter intractability of many parameterized problems. In particular, we define a hierarchy of classes of parameterized problems $F P T \subseteq W[1] \subseteq W[2] \subseteq \cdots \subseteq W[S A T] \subseteq W[P]$ and identify natural complete problems for $W[t]$ for $t \geq 2$. (In other papers we have shown many problems complete for $W[1]$.) DOMINATING SET is shown to be complete for $W[2]$, and thus is not fixed-parameter tractable unless INDEPENDENT SET, CLIQUE, IRREDUNDANT SET, and many other natural problems in $W[2]$ are also fixed-parameter tractable. We also give a compendium of currently known hardness results as an appendix.
\end{abstract}

Key words. fixed-parameter tractable, $W$-hierarchy, parameterized complexity, DOMINATING SET, $t$-NORMALIZED SATISFIABILITY

AMS subject classifications. Primary, 03D15, 68Q15, 68Q25; Secondary, 03D10, 03D20, 03D30, 03D80, $68 \mathrm{R} 10$

1. Introduction. Many natural computational problems have input that consists of a pair of items. For example, the GRAPH GENUS problem is that of determining for an input pair $(G, k)$, where $G$ is a graph and $k$ is a positive integer, whether the graph $G$ embeds on the genus $k$ surface. The problem of MINOR TESTING is that of determining for an input pair of graphs $(G, H)$ whether $H$ is a minor of $G$.

One of the reasons for our interest in parameterized problems is that while many of these problems are $N P$-complete $P S P A C E$-complete, or even provably intractable, it is sometimes the case that only a small range of parameter values are really important in practice, so that the (apparent) intractability of the general problem may be unduly pessimistic information. For many parameterized problems, we now have encouraging and perhaps useful fixed-parameter tractability results, such as the following.

THEOREM 1.1 (Robertson and Seymour [108]). For every fixed graph $H$ it can be determined in time $O\left(n^{3}\right)$ whether a graph $G$ of order $n$ has a minor isomorphic to $H$.

THEOREM 1.2 (Bienstock and Monma [15]). For every fixed $k$, it can be determined in time $O(n)$ whether a graph $G$ of order $n$ can be embedded in the plane so that $k$ faces cover all the vertices.

THEOREM 1.3 (Bodlaender [16]). For every fixed $k$, it can be determined in time $O(n)$ whether a graph $G$ of order $n$ has a spanning tree with at least $k$ leaves.

THEOREM 1.4 (Lagergren [95]). For every fixed $k$, it can be determined in time $O\left(n \log ^{2} n\right)$ whether a graph $G$ of order $n$ has treewidth at most $k$.

THEOREM 1.5 (Plehn and Voigt [102]). For every fixed graph $H$ of treewidth $w$, it can be determined in time $O\left(n^{w+1}\right)$ whether a graph $G$ of order $n$ has a subgraph isomorphic to $H$. (Note that here the parameter is (a coding of) $\mathrm{H}$.)

${ }^{*}$ Received by the editors March 24, 1992; accepted for publication (in revised form) August 1, 1994. Preliminary versions of some of the results of this paper were presented at the 21 st Manitoba Conference on Numerical Mathematics and Computing, Winnipeg, Manitoba, Canada, October 1991. These were reported in [55].

${ }^{\dagger}$ Mathematics Department, Victoria University, P.O. Box 600, Wellington, New Zealand. The research of this author was supported by a grant from Victoria University IGC and by the United States-New Zealand Cooperative Science Foundation grant INT 90-20558.

$\ddagger$ Department of Computer Science, University of Victoria, Victoria, British Columbia, V8W 3P6, Canada. The research of this author was supported by Office of Naval Research contract N00014-88K-0456, National Science Foundation grant MIP-8919312, and the National Science and Engineering Research Council of Canada. 
THEOREM 1.6 (Fellows and Langston [74]). For every fixed $k$, it can be determined in time $O(n)$ whether a graph $G$ of order $n$ has a cycle of length at least $k$.

THEOREM 1.7 (Bodlaender [17], Downey and Fellows [55]). For every fixed $k$, it can be determined in time $O(n)$ whether a graph $G$ of order $n$ contains $k$ vertex-disjoint cycles.

For other parameterized problems, such as DOMINATING SET (given a graph $G$ and a positive integer $k$ is there a set of $k$ vertices in G such that every vertex either belongs to the set or has a neighbour in the set) we have the contrasting situation where essentially no better algorithm is known than the "trivial" one which just exhaustively tries all possible solutions. For each fixed $k, k$-DOMINATING SET is soluable in this way in time $O\left(n^{k+1}\right)$.

We make the following definitions in order to frame these complexity issues.

DEFINITION 1.8. A parameterized problem is a set $L \subseteq \Sigma^{*} \times \Sigma^{*}$ where $\Sigma$ is a fixed alphabet.

For a parameterized problem $L$ and $y \in \Sigma^{*}$ we write $L_{y}$ to denote the associated fixedparameter problem ( $y$ is the parameter) $L_{y}=\{x \mid(x, y) \in L\}$. We refer to $L_{y}$ as the $y$ th slice of $L$.

DEFINITION 1.9. A parameterized problem $L$ is ((weakly) uniformly) fixed-parameter tractable if there exists a constant $\alpha$ and an algorithm to determine if $(x, y)$ is in $L$ in time $f(|y|) \cdot|x|^{\alpha}$, where $f: N \rightarrow N$ is an arbitrary function. If $f$ is recursive then we say that $L$ is strongly uniformly fixed-parameter tractable. Finally, we say that $L$ is nonuniformly f.p. tractable if there is a family of algorithms $\left\{\Psi_{x}: x \in N\right\}$ and a function $f$ such that $\Psi_{x}$ determines if $(x, y)$ is in $L$ in time $f(|y|) \cdot|x|^{\alpha}$.

In recent years a variety of methods useful for demonstrating fixed-parameter tractability have emerged, such as the well-quasiordering results of Robertson and Seymour [106], [107], [108], and general algorithmic methods for bounded treewidth (e.g., Abrahamson and Fellows [4], [5]; Arnborg [7]; Arnborg, Lagergren, and Seese [10]; Bern, Lawler, and Wong [13]; Courcelle [49]; and Wimer, Hedetniemi, and Laskar [118]).

The reader should note an important detail of the definition of fixed-parameter tractability given above. The results of Theorems 1.2-1.7 (and our Theorem 2.1 below) are clearly uniform; the proofs of these results can be implemented as a single algorithm that works for every parameter value. Consider, contrastingly, the consequence of Theorem 1.1 and the graph minor theorem [108] that for each fixed $k$, it can be determined in time $O\left(n^{3}\right)$ whether a graph $G$ of order $n$ embeds on the surface of genus $k$. It is not immediately clear how these (infinitely many) distinct $O\left(n^{3}\right)$ algorithms, each based on a different finite obstruction set, can be combined into a single finite algorithm. This can done, however, by the two different methods of Fellows and Langston [74], [75]. Almost all of the known fixed-parameter tractability results are (or can be made) uniform. While it is possible to construct examples (Downey and Fellows [58]) that show that the notions of tractability are indeed provably distinct, we also remark that there are natural examples of apparently all flavours of tractability. For instance, consider the following examples.

\section{PLANAR IMPROVEMENT}

Instance: A graph $G$.

Parameter: $k$.

Question: Is $G$ a subgraph of a planar graph $G^{\prime}$ of diameter at most $k$ ?

\section{GRAPH LINKING NUMBER}

Instance: A graph $G$.

Parameter: $k$.

Question: Can $G$ be embedded in 3-space so that at most $k$ disjoint cycles are topologically linked? 
We remark that both of these examples are known to be fixed-parameter tractable via the Robertson-Seymour theorems. But at present PLANAR IMPROVEMENT is known only to be weakly uniformly tractable and GRAPH LINKING NUMBER is known only to be nonuniformly tractable.

The difference between the known fixed-parameter complexity of DOMINATING SET and the problems addressed in Theorems 1.1-1.7 and the two examples, is analogous to the apparent complexity difference between $N P$-complete problems and problems in $P$. For most $N P$-complete problems we essentially know no better algorithm than the "trivial" one requiring exponential time which tries all possible solutions.

If $P=N P$ then DOMINATING SET is fixed-parameter tractable. A converse to this statement is not known and is perhaps unlikely. The reasonable (we think) conjecture that DOMINATING SET is not fixed-parameter tractable is thus apparently stronger than the conjecture that $P \neq N P$. Certainly there is oracle evidence to perhaps support this claim (Downey and Fellows [58]). The graph minor theorem has the consequence that for each fixed surface we can decide graph embedability by employing finitely many minor tests. Thus the fixed-parameter tractability of MINOR TESTING leads to the fixed-parameter tractability of the GRAPH GENUS problem. This may be kept in mind as a motivating example for the following definition.

DEFINITION 1.10 (uniform reduction). A (uniform) reduction of a parameterized problem $L$ to a parameterized problem $L^{\prime}$ is an oracle algorithm $A$ that on input $(x, y)$ determines whether $x \in L_{y}$ and satisfies

(1) There is an arbitrary function $f: N \rightarrow N$ and a polynomial $q$ such that the running time of $A$ is bounded by $f(|y|) q(|x|)$.

(2) For each $y \in \Sigma^{*}$ there is a finite subset $J_{y} \subseteq \Sigma^{*}$ such that A consults oracles only for fixed-parameter decision problems $L_{w}^{\prime}$ where $w \in J_{y}$.

Of course in the above an oracle computation takes only one unit of time. If the oracle is consulted only once by $A$, then we will term the reduction many: 1 . As with the notion of tractability, there is a strong version. If the function $f$ and the map taking $y$ to $J_{y}$ are both recursive, we say that the reduction is strongly uniform. (Similarly there is a notion of nonuniform reduction, which we do not consider in detail here.) All of the results we prove in this paper hold for all of the frameworks, with the single exception of Theorem 4.1.

LEMMA 1.11. If the parameterized problem $L$ reduces to the parameterized problem $L^{\prime}$, and if $L^{\prime}$ is fixed-parameter tractable, then $L$ is fixed-parameter tractable.

Proof. Let $f(|y|) q(|x|)$ be the bound on the running time of the reduction from $L$ to $L^{\prime}$, and suppose $L_{w}^{\prime}$ is decidable in time $g(|w|) \cdot n^{\alpha}$. Without loss of generality, we can take $f$ and $g$ to be increasing. Let $y \in \Sigma^{*}$ and let $J_{y} \subseteq \Sigma^{*}$ be the associated finite subset of $\Sigma^{*}$ for the reduction. Then we can determine if $(x, y) \in L$ in time $O\left(f(|y|) q(|x|) g(m)\left(f(|y|) q(|x|)^{\alpha}\right)\right.$ where $m=\max \left\{|w|: w \in J_{y}\right\}$.

Working definition. Actually for the sake of most naturally occuring concrete reductions, we can take a simpler definition than the above. Most concrete reductions are $m$ reductions of the form $(x, k) \mapsto\left(x^{\prime}, f(k)\right)$ with $x^{\prime}$ depending upon $x$ and $k$, running in time $h(k)|x|^{\alpha}$ with $f$ and $h$ recursive. The reason for this is that most natural problems are smooth in the sense that one has a natural encoding of the slices with parameters below $k$ into the $k$ th slice and hence we only need to look at one slice $f(k)$ for any input $(x, k)$. The general definition is useful at times, and is certainly needed for structural theorems. It does the reader no harm to take the simplified definition for the remainder of the paper.

Remark: Alternative definition. Another view of the ideas above is provided by Cai et al. [41], the advice view. In that paper, Cai et al. prove that if $L$ is a parameterized language, 
then $L \in F P T$ iff there is a function $f: N \rightarrow \Sigma^{*}$ (the advice function) and a $P$-time oracle (deterministic) Turing machine $\Phi$, such that for all $(x, k)$,

$$
(x, k) \in L \text { iff } \Phi^{f(k)}(x) \text { accepts. }
$$

That is, if we allow for each $k$ a finite piece of advice, then we can solve all the instances in time $|x|^{\alpha}$. There is similarly a relativized version for the reductions. We refer the reader to Cai et al. [41] for more details.

The plan of this paper is as follows. In $\S 2$ we prove a particular combinatorial problem reduction that plays a key role in our main theorem, which is presented in $\S 3$. Section 4 summarizes our results and discusses open problems. During the time this paper was under consideration many new hardness and completeness results have been found, and we see that this theory seems to have wide-ranging consequences for the classification of parameterized hardness results. This is particularly true of, for instance, problems in molecular biology where one is often interested in a small parameter (e.g., the number of strands of DNA) yet the problem is very large (e.g., the length of the strand), and in very large scale integration design where we might have a small number of wafers of very large size. In the appendix we give a list of currently known hardness results, as well as a list of open classification problems.

Related work and historical remarks. To conclude this section we give some brief remarks concerning related investigations. As far as we are aware, the first person to suggest that something might be interesting in the fact that DOMINATING SET seems to require time $\Omega\left(n^{k+1}\right)$ was Ken Regan [104], in some comments in that paper. Regan did not pursue this issue. There have been investigations into "nondeterminism in $P$ " such as the KintalaFischer $\beta$-hierarchy [93] and the work of Buss and Goldsmith [35] but these and similar related investigations were mainly structural, and looked at problems for a single $k$. One then cannot use $P$-time reductions since the class is in DTIME $\left(n^{f(k)}\right)$ and usually DTIME $\left(n^{k}\right)$. These authors instead used small reductions such as quasilinear (e.g., Gurevich and Shelah [86]) time reductions, which are of course machine dependent. The only paper to truely study the asymptotic parameterized behavior (i.e., the issue of $n^{\alpha}$ versus $n^{f(k)}$ with $f(k) \rightarrow \infty$ ) is Abrahamson et al. [3]. (Some of these results were recast in [35].) In that paper the objects were $P$-checkable, $P$-indexed relations, called (polynomial time) generator tester pairs. For each $k$, one needed to be able to generate a $P$-time list of potential candidates for solutions that were easy to test. The actual definition is very involved but the following example gives the flavour.

If we consider the problem VERTEX COVER, then for an instance $G$ and a parameter $k$, the potential witnesses would be pairs consisting of $G$ and a vertex cover $V$ with the index of $V \leq j=\left(\begin{array}{l}n \\ 0\end{array}\right)+\cdots+\left(\begin{array}{l}n \\ k\end{array}\right)$. The ideas only seemed to apply to relations in NP. While there is a notion of parameterized tractability in [3], it is roughly equivalent to our notion of nonuniform fixed-parameter tractability and hence suffers from the problem that the tractable classes can be nonrecursive. The real problem with that paper is that the notion of reducibility, which is defined on relations rather than parameterized languages, is rather unnatural and very unwieldy, and the notion of intractability is that of (essentially) being $P$-complete (or "dual $P$-complete") under logspace reducibility "by the slice." That is, in [3], problems are $P G T$ complete (in their notation) only when for each $k$ they are more or less $P$-complete. Of course this means that the Abrahamson et al. [3] ideas cannot address things such as DOMINATING SET and INDEPENDENT SET, nor apparently anything in the $W[t]$ classes below. We remark that the Abrahamson et al. [3] results can be easily placed in our setup because they give $W[P]$ completeness results (see, e.g., Abrahamson, Downey, and Fellows [1], [2]). We see our major contributions as identifying the correct notions of reducibility, identifying the correct setting for the study of parameterized intractibility, and finally identifying some "good" problems 
with which to measure hardness. The number of problems that have now been identified as $W[1]$ hard, and hence apparently intractable, would seem to support our claims. (W[1] hardness is not analysed in the present paper, but in Downey and Fellows [56].) At this stage we will only remark that quite a number of parameterized problems have been shown to be $W[1]$-complete. (See the appendix.) We also have a sort of Cook-Levin theorem for $W[1]$ which we feel strongly suggests its intractability. Cai et al. [40] have shown that the following very generic problem is $W[1]$ complete.

\section{SHORT TURING MACHINE ACCEPTANCE}

Input: A nondeterministic Turing machine $M$ and a string $x$.

Parameter: $k$.

Question: Does $M$ have an accepting computation for $x$ in $k$ or fewer steps?

To conclude, we reiterate the remark that the notion of parameterized tractability is not a refinement of the classical notions arising from $N P$-completeness, despite the fact that many of our examples arise from this arena. Problems can be PSPACE complete (e.g., ALTERNATING HITTING SET; see Abrahamson, Downey, and Fellows [2]) and yet have parameterized versions that are $F P T$. Also there are problems that are almost certainly not $N P$-complete unless some unlikely collapse occurs such as $N P$ to $L O G N P$ and yet their parameterized versions can be $W[2]$ or $W[1]$ hard (e.g., the VAPNIK CHERVONENKIS DIMENSION; see Downey, Evans, and Fellows [54], Downey and Fellows [59], and Papadimitriou and Yannakakis [100]). Finally take any set $A$ and consider the parameterized problem $\{(x, x): x \in A\}$. Then this problem is just as hard as $A$ classically, so it can even be provably intractable, and yet it is trivially $F P T$. These examples show that the parameterized complexity of problems and their unparameterized versions are pretty well unrelated, and thus our investigations point to a new dimension in the structure of problems.

2. A key combinatorial reduction. Neither of the well-known computational problems of (1) determining whether a graph $G$ has a dominating set of size $k$ (DOMINATING SET), and (2) determining whether a graph $G$ has an independent set of size $k$ (INDEPENDENT SET) is know to be fixed-parameter tractable, and it is perhaps a reasonable conjecture that they are not. The reader skeptical of this conjecture and willing to challenge it, will be advised by the results of this section to begin by working on INDEPENDENT SET, since a consequence of Theorem 2.1 is that INDEPENDENT SET reduces to DOMINATING SET (and so the latter is "apparently harder" with respect to fixed-parameter tractability). Presently the best known results for these problems are the trivial $O\left(n^{k+1}\right)$ algorithm for DOMINATING SET and a nontrivial algorithm for INDEPENDENT SET due to Nesetril and Poljak [99], requiring time $O\left(n^{k(2+\epsilon) / 3}\right)$, where $2+\epsilon$ represents the best known exponent for fast matrix multiplication.

We show that WEIGHTED CNF SATISFIABILITY (defined below) reduces to DOMINATING SET. By the weight of a truth assignment to a set of boolean variables, we mean the number of variables assigned the value true, in the same way that the weight of a binary vector means the number of 1's in the vector. Since INDEPENDENT SET (and many other parameterized problems) easily reduce to this problem, we have the consequence claimed above. For example, a graph $G=(V, E)$ has a $k$-element independent set if and only if the expression $\prod_{u v \in E}(\bar{u}+\bar{v})$ has a weight $k$ truth assignment. The notion of reduction that we use is (the working reduction) defined in $\S 1$.

\section{WEIGHTED CNF SATISFIABILITY}

Instance: A boolean expression $X$ in conjuctive normal form.

Parameter: $k$.

Question: Is there a truth assignment of weight $k$ that satisfies $X$ ? 


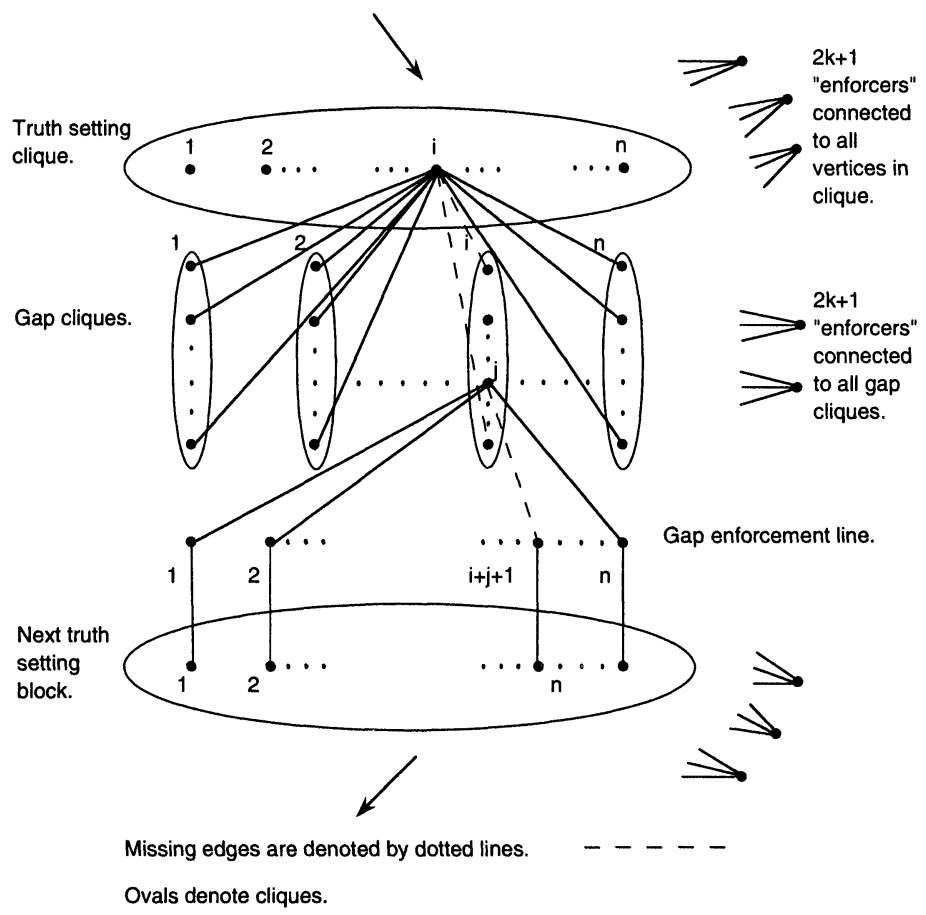

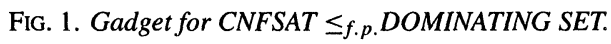

THEOREM 2.1. WEIGHTED CNF SATISFIABILITY strongly uniformly reduces to DOMINATING SET.

Proof. Let $X$ be a Boolean expression in conjuctive normal form consisting of $m$ clauses $C_{1}, \ldots, C_{m}$ over the set of $n$ variables $x_{0}, \ldots, x_{n-1}$. We show how to produce in polynomialtime by local replacement a graph $G=(V, E)$ that has a dominating set of size $2 k$ if and only if $X$ is satisfied by a truth assignment of weight $k$.

A diagram of the gadget used in the reduction is given in Fig. 1. The idea of the proof is as follows. There are $k$ of the gadgets arranged in a circle. Each of the gadgets has three main parts. Taken clockwise from top to bottom, these are variable selection, gap selection, and gap enforcement. The variable selection component is a clique and the gap selection consists of $n$ cliques which we call columns. Our first action is to ensure that in any dominating set of $2 k$ elements, we must pick one vertex from each of these two components. This goal is achieved by the $2 k$ sets of $2 k+1$ enforcers, vertices from $V_{4}$ and $V_{5}$. (The names refer to the sets below.) Take the set $V_{4}$, for instance. For a fixed $r$, these $2 k+1$ vertices are connected to all of the variable selection vertices in the component $A(r)$, and nowhere else. Thus if they are to be dominated by a $2 k$ dominating set, then we must choose some element in the set $A(r)$, and similarly we must choose an element in the set $B(r)$ by virtue of the $V_{5}$ enforcers. Since we will need exactly $2 k$ (or even $\leq 2 k$ ) dominating elements it follows that we must pick exactly one from each of the $A(r)$ and $B(r)$ for $r=1, \ldots, k$.

As the name suggests these will be picked by the variable selection components, $A(r)$, $r=0, \ldots, k-1$. Each of these $k$ components consists of a clique of $n$ vertices labeled $0, \ldots, n-1$, the intention being that the vertex labeled $i$ represents a choice of variable $i$ being made true in the formula $X$. Correspondingly in the next $B(r)$ we have columns (cliques) $i=0, \ldots, n-1$. The intention is that column $i$ corresponds to the choice of variable $i$ in 
the preceding $A(r)$. The idea then is the following. We join the vertex $a[r, i]$ corresponding to variable $i$, in $A(r)$, to all vertices in $B(r)$ except those in column $i$. This means that the choice of $i$ in $A(r)$ will cover all vertices of $B(r)$ except those in this column. It follows that we must choose the dominating element from this column and nowhere else. (There are no connections from column to column.) The columns are meant to be the gap selection that says how many 0 's there will be until the next positive choice for a variable. We finally need to ensure that if we chose variable $i$ in $A(r)$ and gap $j$ in column $i$ from $B(r)$ then we need to pick $i+j+1$ in $A(r+1)$. This is the role of the gap enforcement component which consists of a set of $n$ vertices (in $V_{6}$.) The method is to connect vertex $j$ in column $i$ of $B(r)$ to all of the $n$ vertices $d[r, s]$ except to $d[r, i+j+1]$. The point of this is that if we choose $j$ in column $i$ we will dominate all of the $d[r, s]$ except $d[r, i+j+1]$. Since we will only connect $d[r, s]$ additionally to $a[r+1, s]$ and nowhere else, to choose an element of $A[r+1]$ and still dominate all of the $d[r, s]$ we must actually choose $a[r+1, i+j+1]$.

Thus the above provides a selection gadget that chooses $k$ true variables with the gaps representing false ones. We enforce that the selection is consistent with the clauses of $X$ via the clause variables $V_{3}$. These are connected in the obvious ways. One connects a choice in $A[r]$ or $B[r]$ corresponding to making a clause $C_{q}$ true to the variable $c_{q}$. Then if we dominate all the clause variables too, we must have either chosen in some $A[r]$ a positive occurrence of a variable in $C_{q}$ or we must have chosen in $B[r]$ a gap corresponding to a negative occurrence of a variable in $C_{q}$, and conversely. We now turn to the formal details.

The vertex set $V$ of $G$ is the union of the following sets of vertices:

$$
\begin{aligned}
& V_{1}=\{a[r, s]: 0 \leq r \leq k-1,0 \leq s \leq n-1\}, \\
& V_{2}=\{b[r, s, t]: 0 \leq r \leq k-1,0 \leq s \leq n-1,1 \leq t \leq n-k+1\}, \\
& V_{3}=\{c[j]: 1 \leq j \leq m\}, \\
& V_{4}=\left\{a^{\prime}[r, u]: 0 \leq r \leq k-1,1 \leq u \leq 2 k+1\right\}, \\
& V_{5}=\left\{b^{\prime}[r, u]: 0 \leq r \leq k-1,1 \leq u \leq 2 k+1\right\}, \\
& V_{6}=\{d[r, s]: 0 \leq r \leq k-1,0 \leq s \leq n-1\} .
\end{aligned}
$$

For convenience, we introduce the following notation for important subsets of some of the vertex sets above. Let

$$
\begin{aligned}
A(r) & =\{a[r, s]: 0 \leq s \leq n-1\}, \\
B(r) & =\{b[r, s, t]: 0 \leq s \leq n-1,1 \leq t \leq n-k+1\}, \\
B(r, s) & =\{b[r, s, t]: 1 \leq t \leq n-k+1\} .
\end{aligned}
$$

The edge set $E$ of $G$ is the union of the following sets of edges. In these descriptions we implicitly quantify over all possible indices.

$$
\begin{aligned}
& E_{1}=\left\{c[j] a[r, s]: x_{s} \in C_{j}\right\}, \\
& E_{2}=\left\{a[r, s] a\left[r, s^{\prime}\right]: s \neq s^{\prime}\right\}, \\
& E_{3}=\left\{b[r, s, t] b\left[r, s, t^{\prime}\right]: t \neq t^{\prime}\right\}, \\
& E_{4}=\left\{a[r, s] b\left[r, s^{\prime}, t\right]: s \neq s^{\prime}\right\}, \\
& E_{5}=\left\{b[r, s, t] d\left[r, s^{\prime}\right]: s^{\prime} \neq s+t(\bmod n)\right\}, \\
& E_{6}=\left\{a[r, s] a^{\prime}[r, u]\right\}, \\
& E_{7}=\left\{b[r, s, t] b^{\prime}[r, u]\right\}, \\
& E_{8}=\left\{c[j] b[r, s, t]: \exists i \overline{x_{i}} \in C_{j}, s<i<s+t\right\}, \\
& E_{9}=\left\{d[r, s] a\left[r^{\prime}, s\right]: r^{\prime}=r+1(\bmod n)\right\} .
\end{aligned}
$$


Suppose $X$ has a satisfying truth assignment $\tau$ of weight $k$, with variables $x_{i_{0}}, x_{i_{1}}, \ldots, x_{i_{k-1}}$ assigned the value true. Suppose $i_{0}<i_{2}<\cdots<i_{k-1}$. Let $d_{r}=i_{r+1(\bmod k)}-i_{r}(\bmod n)$ for $r=0, \ldots, k-1$. It is straightforward to verify that the set of $2 k$ vertices

$$
D=\left\{a\left[r, i_{r}\right]: 0 \leq r \leq k-1\right\} \cup\left\{b\left[r, i_{r}, d_{r}\right]: 0 \leq r \leq k-1\right\}
$$

is a dominating set in $G$.

Conversely, suppose $D$ is a dominating set of $2 k$ vertices in $G$. The closed neighborhoods of the $2 k$ vertices $a^{\prime}[0,1], \ldots, a^{\prime}[k-1,1], b^{\prime}[0,1], \ldots, b^{\prime}[k-1,1]$ are disjoint, so $D$ must consist of exactly $2 k$ vertices, one in each of these closed neighborhoods. Also, none of the vertices of $V_{4} \cup V_{5}$ are in $D$, since if $a^{\prime}[r, u] \in D$ then necessarily $a^{\prime}\left[r, u^{\prime}\right] \in D$ for $1<u^{\prime}<2 k+1$ (otherwise $D$ fails to be dominating), which contradicts that $D$ contains exactly $2 k$ vertices. It follows that $D$ contains exactly one vertex from each of the sets $A(r)$ and $B(r)$ for $0 \leq r \leq k-1$.

The possibilities for $D$ are further constrained by the edges of $E_{4}, E_{5}$, and $E_{9}$. The vertices of $D$ in $V_{1}$ represent the variables set to true in a satisfying truth assignment for $X$, and the vertices of $D$ in $V_{2}$ represent intervals of variables set to false. Since there are $k$ variables to be set to true there are, considering the indices of the variables $\bmod n$, also $k$ intervals of variables to be set to false.

The edges of $E_{4}, E_{5}$, and $E_{9}$ enforce that the $2 k$ vertices in $D$ must represent such a choice consistently. To see how this enforcement works, suppose $a[3,4] \in D$. This represents that the third of $k$ distinct choices of variables to be given the value true is the variable $x_{4}$. The edges of $E_{4}$ force the unique vertex of $D$ in the set $B(3)$ to belong to the subset $B(3,4)$. The index of the vertex of $D$ in the subset $B(3,4)$ represents the difference $(\bmod n)$ between the indices of the third and fourth choices of a variable to receive the value true, and thus the vertex represents a range of variables to receive the value false. The edges of $E_{5}$ and $E_{9}$ enforce that the index $t$ of the vertex of $D$ in the subset $B(3,4)$ represents the "distance" to the next variable to be set true, as it is represented by the unique vertex of $D$ in the set $A(4)$.

It remains only to check that the fact that $D$ is a dominating set ensures that the truth assignment represented by $D$ satisfies $X$. This follows by the definition of the edge sets $E_{1}$ and $E_{8}$.

Because DOMINATING SET can be easily reduced to WEIGHTED CNF SATISFIABILITY with no negated literals, the above theorem shows the surprising fact that WEIGHTED SATISFIABILITY reduces to MONOTONE WEIGHTED CNF SATISFIABILITY. (The reduction is straightforward. Let $\left\{x_{1}, \ldots, x_{n}\right\}$ denote the set of vertices of the given graph $G$, and we will interpret them as input variables for our circuit $C(G)$. Have a layer of $O r$ gates directly below the variables. These are also one per input variable and we will label them $g_{1}, \ldots, g_{n}$. Make $x_{i}$ and input to the $O r$ gate $g_{j}$ precisely in the case that $\left(x_{i}, x_{j}\right)$ is an edge of $G$. Now to complete the circuit, we have one large And gate with inputs from each of the Or gates. It is easy to see that satisfying assignments correspond directly to dominating sets and conversely.) Interpreted in terms of circuits, this combinatorial reduction plays a crucial role in the fundamental completeness results surveyed in the next section. We can use this reduction in the main theorem (Theorem 3.7) because the reduction proves rather more than is stated in Theorem 2.1 and the relavant properties we need are stated in the remark below. (It is also used to generalize Theorem 2.1 to MONOTONE $2 t$-NORMALIZED BOOLEAN FORMULAE in Corollary 3.8.)

Remark. An important fact about the above proof is the following. For our fixed $k$ the enforcement gadgetry causes us to choose the $2 k$ vertices, $k$ from the $A(r)$ 's and $k$ from the $B$ 's, and there is a 1-1 correspondence between weight $k$ assignments to $X$ and size $2 k$ sets that dominate the graph $G^{\prime}$ which denotes the gadget, that is, $G$ without the clause 
connections and vertices. Define a weak dominating set to be a set of $(2 k)$ vertices that dominates the gadget of $G$. The fact we will use in the proof of the normalization theorem is that under the 1-1 correspondence between weight $k$ assignments to $X$ and weak dominating sets, if a clauses $C_{i_{1}}, \ldots, C_{i_{p}}$ are the subset of the set of clauses satisfied by some weight $k$ assignment, then $c_{i_{1}}, \ldots, c_{i_{p}}$ are exactly the clause vertices dominated by the corresponding size $2 k$ weak dominating set in $G$. That is, not only is there a correspondence between weight $k$ satisfying assignments for $X$ and weight $2 k$ dominating sets in $G$, but in fact there is an exact correspondence between all weight $k$ assignments together with the clauses they satisfy, and with all weak dominating sets and the clause vertices they dominate.

The DOMINATING SET reduction also allows for a number of other applications. For instance, consider the problem below.

\section{WEIGHTED $\{0,1\}$-INTEGER PROGRAMMING}

Instance: A binary matrix $A$ and a binary vector $\mathbf{b}$.

Parameter: $k$.

Question: Does $A \cdot \mathbf{x} \geq \mathbf{b}$ have a binary solution of weight $k$ ?

We have the following corollary.

COROLLARY 2.2. WEIGHTED CNF SATISFIABILITY reduces to WEIGHTED $\{0,1\}$ INTEGER PROGRAMMING.

Proof. Let $(X, k)$ be an instance of MONOTONE WEIGHTED CNF SAT. Let $C_{1}, \ldots, C_{p}$ list the clauses of $X$ and $x_{1}, \ldots, x_{m}$ list the variables. Let $A$ be the matrix $\left\{a_{i, j}: i=\right.$ $1, \ldots, p, j=1, \ldots, m\}$ with $a_{i, j}=1$ if $x_{j}$ is present in $C_{i}$ and $a_{i, j}=0$ otherwise. Let $\mathbf{b}$ be the vector with 1 in the $j$ th position for $j=1, \ldots, p$. It is easy to see that $A \cdot \mathbf{x} \geq \mathbf{b}$ has a solution of weight $k$ iff $X$ has a satisfying assignment of weight $k$ (and the reasoning is reversible).

In passing we remark that the situation of Corollary 2.2 , where a classical reduction can easily be modified for a normal parameterized case, is exceedingly rare. It seems to be that it is almost never the case that a classical reduction gives rise to a parameterized one.

3. A completeness theory for fixed-parameter intractability. In order to frame a completeness theory to address the apparent fixed-parameter intractability of DOMINATING SET and other problems, we need to define appropriate classes of parameterized problems. As one might expect, satisfiability occupies a central role in our investigations. But now the situation is apparently much more complex than in the classical case. For instance, while classically there is no difference between CNF SATISFIABILITY and SATISFIABILITY for general formulae in propositional logic, there seems to be no parameterized reduction computing general SATISFIABILITY from CNF SATISFIABILITY and hence they do seem to be genuinely of different complexity from a parameterized point of view. Considerations such as these lead to the conclusion that there seem to be many different parameterized degree classes of natural problems. This is quite different from the situation in classical, say, $N P$-completeness results where virtually all natural problems which are not in $P$ seem to be NP complete.

Current measure of intractability. At the present time if we wish to prove parameterized intractability, we show that the problem at hand can compute WEIGHTED 3CNF SATISFIABILITY. This is the class we call $W[1]$ and as we said before, we seem to have very strong evidence that it is intractable. We refer the reader to Downey and Fellows [56].

$t$-normalized formulae. In fact, there does not seem to be any reduction from parameterized CNF to $3 \mathrm{CNF}$. This leads naturally to the realization that the logical depth of a propositional formula affects its parameterized complexity. Thus if CNF is thought of as products-of-sums of literals, then we can define a propositional formula to be $t$-normalized if it is of the form products-of-sums-of-products ... of literals with $t$-alternations. (Hence 
2-normalized is the same as CNF.) Now we believe that for all $t$, WEIGHTED SATISFIABILITY for $t$-normalized formulae is strictly easier than WEIGHTED SATISFIABILITY for $t+1$-normalized formulae. This suggests a hierarchy based on the complexity of $t$-normalized formulae. It turns out that this hierarchy is quite useful for the classification of the complexity of many natural problems, and $t$-normalized formulae do give quite a bit more computational power. We make this more precise through the introduction of the circuit-based classes below.

The classes that we define below are intuitively based on the complexity of the circuits required to check a solution. The size of a circuit is as usual the number of gates in the circuit.

We first define decision circuits in which some gates have bounded fan-in and some have unrestricted fan-in. It is assumed that fan-out is never restricted.

DEFINITION 3.1. A circuit is of mixed type if it consists of circuits having gates of the following kinds:

(1) Small gates: not gates, and gates and or gates with bounded fan-in. We will usually assume that the bound on fan-in is 2 for and gates and or gates, and 1 for not gates.

(2) Large gates: And gates and Or gates with unrestricted fan-in.

We will use lowercase to denote small gates (or gates and and gates), and uppercase to denote large gates (Or gates and And gates).

DEFINITION 3.2 (depth and weft). The depth of a circuit $C$ is defined to be the maximum number of gates (small or large) on an input-output path in $C$. The weft of a circuit $C$ is the maximum number of large gates on an input-output path in $C$.

DEFINITION 3.3. We say that a family of circuits $\mathcal{F}$ has bounded depth if there is a constant $h$ such that every circuit in the family $\mathcal{F}$ has depth at most $h$. We say that $\mathcal{F}$ has bounded weft if there is constant $t$ such that every circuit in the family $\mathcal{F}$ has weft at most $t . \mathcal{F}$ is monotone if the circuits of $\mathcal{F}$ do not have not-gates. A circuit $C$ is a decision circuit if it has a single output. A decision circuit $C$ accepts an input vector $x$ if the single output gate has value 1 on input $x$.

DEFINITION 3.4. Let $\mathcal{F}$ be a family of decision circuits. We allow that $\mathcal{F}$ may have many different circuits with a given number of inputs. To $\mathcal{F}$ we associate the parameterized circuit problem $L_{\mathcal{F}}=\{(C, k): C \in \mathcal{F}$ accepts an input vector of weight $k\}$.

DEFINITION 3.5. A parameterized problem $L$ belongs to $W[t]$ (monotone $W[t]$ ) if $L$ uniformly $(m-)$ reduces to the parameterized circuit problem $L_{\mathcal{F}}$ for the family $\mathcal{F}_{h, t}$ of depth $h$, mixed type (monotone) decision circuits of weft at most $t$.

DEFINITION 3.6. We denote the class of fixed-parameter tractable problems as FPT.

Thus we have the containments

$$
F P T \subseteq W[1] \subseteq W[2] \subseteq \cdots
$$

and we conjecture that each of these containments is proper. We term the union of these classes together with two other classes $W[S A T] \subseteq W[P]$, the $W$-Hierarchy. Here $W[P]$ denotes the class obtained by having no restriction on depth, i.e., $P$-size circuits, and $W[S A T]$ denotes the restriction to boolean circuits of $P$-size. We do not explore $W[S A T]$ or $W[P]$ here but do so in Abrahamson, Downey, and Fellows [1], [2].

Our main result shows that WEIGHTED CNF SATISFIABILITY is complete for $W[2]$ and that similar problems are complete for each level of the $W$-Hierarchy of parameterized problem classes. This theorem, Theorem 3.7, plays a role in our theory analogous to Cook's theorem for $N P$-completeness, in the following sense. Usually proofs of membership in a particular $W[t]$ class are easy, so the circuit definition is easy to reduce $t o$, whereas the $t$ normalized formulae provide problems that are easy to reduce from to establish hardness. The other view of the Cook(-Levin) theorem is that it connects a generic problem from Turing machines to SATISFIABILITY and hence SATISFIABILITY is very unlikely to be tractable. 
This other view of the Cook(-Levin) theorem is not pursued here but is established for $W[1]$ in Downey and Fellows [56], as we mentioned earlier. The present paper, and particularly Theorem 3.7, provide an important technical framework for hardness results.

It is interesting that the combinatorial reduction of Theorem 2.1, and the subsequent remark play a key role (as a "change of variables") in our proof of Theorem 3.7. Thus the entire argument that DOMINATING SET is complete for $W[2]$ actually uses this combinatorial reduction twice. Recall the notion of $t$-normalized formula we discussed earlier. It naturally gives rise to the following problem.

\section{WEIGHTED $t$-NORMALIZED SATISFIABILITY}

Input: A $t$-normalized boolean expression $X$.

Parameter: A positive integer $k$.

Question: Does $X$ have a satisfying truth assignment of weight $k$ ?

THEOREM 3.7. For $t \geq 2$ WEIGHTED $t$-NORMALIZED SATISFIABILITY is many: 1 complete for $W[t]$.

Proof. Let $L \in W[t]$. Let $\mathcal{F}$ be the family of circuits of depth bounded by $h$ and weft bounded by $t$ to which $L$ reduces. It suffices to reduce $L_{\mathcal{F}}$ to WEIGHTED $t$-NORMALIZED SATISFIABILITY. An instance of the latter problem may be viewed as a pair consisting of a positive integer $k$ and a circuit having $t$ alternating layers of And and $O r$ gates corresponding to the $t$-normalized expression structure P-o-S-o-P- ..., and having a single output And gate. Thus the argument essentially shows how to "normalize" the circuits in $\mathcal{F}$.

Let $(C, k)$ be an instance of $L_{\mathcal{F}}$. We show how to determine whether $C$ accepts a weight $k$ input vector by consulting an oracle for WEIGHTED $t$-NORMALIZED SATISFIABILITY (viewed as a problem about circuits) for finitely many weights $k^{\prime}$. The algorithm for this determination will be uniform in $k$, and run in time $f(k) n^{\alpha}$ where $n$ is the size of the circuit $C$. The exponent $\alpha$ will be a (possibly exponential) function of $h$ and $t$. This is permissible, since every circuit in $\mathcal{F}$ observes these bounds on depth and weft.

Step 1. The reduction to tree circuits.

The first step is to transform $C$ into a tree circuit $C^{\prime}$ (or formula) of depth and weft bounded by $h$ and $t$, respectively. In a tree circuit every logic gate has fan-out one. (The input nodes may have large fan-out.) The transformation is accomplished by replicating the portion of the circuit above a gate as many times as the fan-out of the gate, beginning with the top level of logic gates and proceeding downward level by level. (We regard a decision circuit as arranged with the inputs on top and the output on the bottom.) The creation of $C^{\prime}$ from $C$ may require time $O\left(n^{O(h)}\right)$ and involve a similar blow-up in the size of the circuit. The tree circuit $C^{\prime}$ accepts a weight $k$ input vector if and only if the original circuit $C$ accepts a weight $k$ input vector.

Step 2. Moving the not gates to the top of the circuit.

Let $C$ denote the circuit we receive from the previous step (we will use this notational convention throughout the proof). Transform $C$ into an equivalent circuit $C^{\prime}$ by commuting the not gates to the top, using DeMorgan's laws. This may increase the size of the circuit by at most a constant factor. The tree circuit $C^{\prime}$ thus consists (from the top) of the input nodes, with not gates on some of the lines fanning out from the inputs. In counting levels we consider all of this as level 0 , and may refer to negated fan-out lines from the input nodes as negated inputs. Next, there are levels consisting only of large and small and and or gates, with a single output gate (which may be of either principal logical denomination at this point).

Step 3. Homogenizing the layers.

The goal of this step is to reduce to the situation where all of the large gates are at the bottom of the circuit, in alternating layers of large And and $O r$ gates. To achieve this we work from the bottom up, with the first task being to arrange for the output gate to be large. 
Let $C$ denote the circuit received from the previous step. Suppose the output gate $z$ is small. Let $C[z]$ denote the connected component of $C$ including $z$ that is induced by the set of small gates. Thus all gates providing input to $C[z]$ are either large or are input gates of $C$. Because of the bound $h$ on the depth of $C$, there are at most $2^{h}$ inputs to $C[z]$. The function of these inputs computed by $C[z]$ is equivalent to a product-of-sums expression $E_{z}$ having at most $2^{2^{h}}$ sums, with each sum a product of at most $2^{h}$ inputs. Let $C^{\prime}$ denote the circuit equivalent to $C$ obtained by replacing the small gate output component $C[z]$ with $E_{z}$, duplicating subcircuits of $C$ as needed to provide the inputs to the depth 2 circuit representing $E_{z}$. (The "product" gate of $E_{z}$ is now the output gate of $C^{\prime}$.) This entails a blow-up in size by a factor bounded by $2^{2^{h}}$. Since $h$ is an absolutely fixed constant (not dependent on $n$ or $k$ ) this blow-up is "linear" and permitted. Note that $E_{z}$ and therefore $C^{\prime}$ are easily computed in a similar amount of time to this size blow-up.

Let $p$ denote the output and gate of $C^{\prime}$ (corresponding to the product in $E_{z}$ ). Let $s_{1}, \ldots, s_{m}$ denote the or gates of $C^{\prime}$ corresponding to the sums of $E_{z}$. We consider all of these gates to be small, since the number of inputs to them does not depend on $n$ or $k$. (Equivalently, if the gates of these two levels were replaced by binary input gates, we would see that the reduction of $C$ to $C^{\prime}$ has increased circuit depth from $h$ to $2^{h}$.)

Each or gate $s_{i}$ of $C^{\prime}$ has three kinds of input lines: those coming from large And gates, those coming from large $O r$ gates, and those coming from input gates of $C^{\prime}$. We will use the same symbol to denote an input line, the subcircuit of $C^{\prime}$ that computes the value on that line, or the Boolean expression corresponding to the subcircuit (since $C^{\prime}$ is a tree circuit, it is equivalent to a Boolean expression). Let these three groups of inputs be denoted, respectively, by

$$
\begin{aligned}
& S_{i, \wedge}=\left\{s_{i}[\wedge, j]: j=1, \ldots, m_{i, \wedge}\right\}, \\
& S_{i, \vee}=\left\{s_{i}[\vee, j]: j=1, \ldots, m_{i, \vee}\right\}, \\
& S_{i, \top}=\left\{s_{i}[\top, j]: j=1, \ldots, m_{i, \top}\right\},
\end{aligned}
$$

and define

$$
S_{i}=S_{i, \wedge} \cup S_{i, \vee} \cup S_{i, \top}
$$

For each line $s_{i}[\vee, j]$ of $C^{\prime}$ coming from a large Or gate $u$, let

$$
S_{i, \vee, j}=\left\{s_{i}[\vee, j, k]: k=1, \ldots, m_{i, \vee, j}\right\}
$$

denote the set of input lines to $u$ in $C^{\prime}$. Similarly, for each line $s_{i}[\wedge, j]$ of $C^{\prime}$ coming from a large And gate $v$, let

$$
S_{i, \wedge, j}=\left\{s_{i}[\wedge, j, k]: k=1, \ldots, m_{i, \wedge, j}\right\}
$$

denote the set of input lines to $v$ in $C^{\prime}$.

Let

$$
k^{\prime}=\sum_{i=1}^{m}\left(1+m_{i, \vee}\right)
$$

The integer $k^{\prime}$ is the number of or gates (counting both large and small gates) that are either part of $C[z]$ or directly supply input to $C[z]$. Note that $k^{\prime}$ is bounded above by $2^{h} \cdot 2^{2^{h}}$. 
We describe how to produce a weft $t$ circuit $C^{\prime \prime}$ from $C^{\prime}$ that accepts an input vector of weight $k^{\prime \prime}=k+k^{\prime}$ if and only if $C^{\prime}$ (and therefore $C$ ) accepts an input vector of weight $k$. The tree circuit $C^{\prime \prime}$ will have a large And gate giving the output.

Let $x_{1}, \ldots, x_{n}$ denote the inputs to $C^{\prime}$. The circuit $C^{\prime \prime}$ has additional input variables that, for the most part, correspond to the input lines to the or gates singled out for attention above. The set $V$ of new input variables is the union of the following groups of variables:

$$
V=\left(\bigcup_{i=1}^{m} V_{i}\right) \cup\left(\bigcup_{i=1}^{m} \bigcup_{j=1}^{m_{i, \vee}} V_{i, j}\right) \text {, }
$$

where

$$
V_{i}=\left\{v_{i}[\wedge, j]: 1 \leq j \leq m_{i, \wedge}\right\} \cup\left\{v_{i}[\vee, j]: 1 \leq j \leq m_{i, \vee}\right\} \cup\left\{v_{i}[\top, j]: 1 \leq j \leq m_{i, \top}\right\}
$$

and

$$
V_{i, j}=\left\{v_{i}[\vee, j, k]: 1 \leq k \leq m_{i, \vee, j}\right\} \cup\{n[i, j]\} .
$$

The circuit $C^{\prime \prime}$ is represented by the Boolean expression

$$
C^{\prime \prime}=E_{1} \cdot E_{2} \cdot E_{3} \cdot E_{4} \cdot E_{5} \cdot E_{6} \cdot E_{7},
$$

where

$$
\begin{gathered}
E_{1}=\prod_{i=1}^{m}\left(\sum_{u \in V_{i}} u\right), \\
E_{2}=\prod_{i=1}^{m} \prod_{u \neq v, u, v \in V_{i}}(\neg u+\neg v), \\
E_{4}=\prod_{i=1}^{m} \prod_{j=1}^{m_{i, \vee}} u, v \in V_{i, j}^{m}(\neg u+\neg v), \\
E_{5}=\prod_{i=1}^{m} \prod_{j=1}^{m_{i, v}}\left(\sum_{j=1}^{m_{i, \wedge}} u\right), \\
E_{7} \prod_{k=1}^{m_{i, \wedge, j}}\left(s_{i}[\wedge, j, k]+\neg v_{i}[\wedge, j]\right), \\
E_{6}=\prod_{i=1}^{m} \prod_{j=1}^{m} \prod_{j=1}^{m_{i, v}} \prod_{k=1}^{m_{i, v}}\left(\neg v_{i}[\vee, j]+\neg n[i, j]\right),
\end{gathered}
$$


The size of $C^{\prime \prime}$ is bounded by $\left|C^{\prime}\right|^{2}$.

Claim 1. The circuit $C^{\prime \prime}$ has weft $t$.

To see this, note first that since $t \geq 2$, any input-output path beginning from a new input variable (in $V$ ) that has at most two large gates as the expression for $C^{\prime \prime}$ is essentially a product-of-sums. In $E_{5}$ and $E_{7}$ the sums involve subexpressions of $C^{\prime}$; any input-output path from an original input variable (of $C^{\prime}$ ) passes through one of these or gates. Observe that in $C^{\prime}$ these subexpressions have weft at most $t-1$. The sums of $E_{5}$ and $E_{7}$ are small, so these paths further encounter only the bottommost large And gate.

Claim 2. The circuit $C^{\prime \prime}$ accepts an input vector of weight $k^{\prime \prime}$ if and only if $C^{\prime}$ accepts an input vector of weight $k$.

First note that any input vector of weight $k^{\prime \prime}$ accepted by $C^{\prime \prime}$ must set exactly one variable in each of the sets of variables $V_{i}$ (for $i=1, \ldots, m$ ) and $V_{i, j}$ (for $i=1, \ldots, m$ and $j=$ $\left.1, \ldots, m_{i, \vee}\right)$ to 1 and all of the others in the set to 0 in order to satisfy $E_{1} \cdot E_{2} \cdot E_{3} \cdot E_{4}$. It follows that any such accepted input must set exactly $k$ of the original variables of $C^{\prime}$ to 1 , by the definition of $k^{\prime \prime}$.

The role of the (new) variables set to 1 in the sets of variables that represent inputs to or gates is to indicate an accepting computation of $C^{\prime}$ on the weight $k$ input of old variables. The expressions $E_{5}, \ldots, E_{7}$ enforce the correctness of this representation in $C^{\prime \prime}$ of the computation of $C^{\prime}$.

The expression $E_{5}$ ensures that if the new variable $v_{i}[\wedge, j]$ is set to 1 , indicating that the subexpression $s_{i}[\wedge, j]$ of $C^{\prime}$ evaluates to 1 , then every argument $s_{i}[\wedge, j, k]$ must evaluate to 1 . (Note that subexpressions $s_{i}[\wedge, j, k]$ appear in $C^{\prime \prime}$ while the subexpressions $s_{i}[\wedge, j]$ do not. The computations performed in $C^{\prime}$ by the latter are simply represented by the values of the input variables in $V$.) The role of the variables $n[i, j]$ is to represent that "none of the inputs" to the or gate has the value 1 . The expression $E_{6}$ enforces that if this situation is represented, then the output of the gate is not represented as having the value 1 . The expression $E_{7}$ ensures that if the new variable $v_{i}[\vee, j, k]$ has the value 1 , indicating that the subexpression $s_{i}[\vee, j, k]$ of $C^{\prime}$ evaluates to 1 , then this subexpression must in fact evaluate to 1 .

By the above, we may now assume that the circuit we are working with has a large output gate (which may be of either denomination). Renaming for convenience, let $C$ denote the circuit we are working with under this assumption.

If $g$ and $g^{\prime}$ are gates in $C$ of the same logical character $(\wedge$ or $\vee$ ) with the output of $g$ going to $g^{\prime}$, then they can be consolidated into a single gate without increasing weft if $g$ is small and $g^{\prime}$ is large. We term this a permitted contraction. Note that if $g$ is large and $g^{\prime}$ is small then contraction may not preserve weft. We will assume that permitted contractions are performed whenever possible, interleaved with the following two operations.

(1) Replacement of bottommost small gate components.

As at the beginning of Step 3, let $C_{1}, \ldots, C_{m}$ denote the bottommost connected components of $C$ induced by the set of small gates and having at least one large gate input. Since the output gate of $C$ is large, each $C_{i}$ gives output to a large gate $g_{i}$. If $g_{i}$ is an And gate, then $C_{i}$ should be replaced with product-of-sums circuitry equivalent to $C_{i}$. If $g_{i}$ is an $\mathrm{Or}$ gate, then $C_{i}$ should be replaced with equivalent sum-of-products circuitry. Note that in either case this immediately creates the opportunity for a permitted contraction. As per the discussion at the beginning of Step 3, this replacement circuitry is small, and this operation may increase the size of the circuit by a factor of $2^{2^{h}}$. This step will be repeated at most $h$ times, as we are working from the bottom up in transforming $C$.

(2) Commuting small gates upward.

After (1), and after the permitted contractions, the bottommost small gate components are each represented in the modified circuit $C^{\prime}$ by a single small gate $h_{i}$ giving output to $g_{i}$. 
Without loss of generality, all of the arguments to $h_{i}$ may be considered to come from large gates. (The only other possibility is that an input argument to $h_{i}$ may be from the input level of the circuit, but there is no increase in weft in treating this as an honorary large gate for convenience.) Suppose that $g_{i}$ is an And gate and that $h_{i}$ is an or gate (the other possibility is handled dually).

There are three possible cases:

(i) All of the arguments to $h_{i}$ are from $O r$ gates.

(ii) All of the arguments to $h_{i}$ are from And gates.

(iii) The arguments to $h_{i}$ include both large $O r$ gates and large And gates.

In case (i), we may consolidate $h_{i}$ and all of the gates giving input to $h_{i}$ into a single large Or gate without increasing weft.

In case (ii), we replace the small $\vee\left(h_{i}\right)$ of large $\wedge$ 's with the equivalent (by distribution) large $\wedge$ of small $\vee$ 's. Since $h_{i}$ may have $2^{h}$ inputs, this may entail a blow-up in the size of the circuit from $n$ to $n^{2^{h}}$. This does not increase weft, and creates the opportunity for a permitted contraction.

In case (iii), we similarly replace $h_{i}$ and its argument gates with circuitry representing a product-of-sums of the inputs to the arguments of $h_{i}$. The difference is that in this case, the replacement is a large $\wedge$ of large (rather than small) $\vee$ gates. Weft is preserved when we take advantage of the contraction now permitted between the large $\wedge$ gate and $g_{i}$.

We may achieve our purpose in this step by repeating the cycle of (1) and (2). At most $h$ repetitions are required. The total blow-up in the size of the circuit in this step is crudely bounded by $n^{2^{h^{2}}}$.

Step 4. Removing a bottommost Or gate.

By a Turing reduction, we can determine whether a tree circuit giving output from an $\mathrm{Or}$ gate accepts a weight $k$ input vector by simply making the same determination for each of the input branches (subformulae) to the gate.

In order to accomplish this step by a many: 1 reduction, we do the following. Let $b$ be the number of branches of the circuit $C$ with bottommost $O r$ gate that we receive at the beginning of this step. We modify $C$ by creating new inputs $x[1], \ldots, x[b]$. The purpose of these input variables is to indicate which branch of $C$ accepts a weight $k$ input vector. Let $C_{1}, \ldots, C_{b}$ be the branches of $C$, so that $C$ is represented by the expression $C_{1}+\cdots+C_{b}$. The modified circuit $C^{\prime}$ is represented by the expression

$$
C^{\prime}=(x[1]+\cdots+x[b]) \cdot \prod_{1 \leq i<j \leq b}(\neg x[i]+\neg x[j]) \cdot \prod_{1 \leq i \leq b}\left(C_{i}+\neg x[i]\right) .
$$

The first two product terms of the above expression ensure that exactly one of the new variables must have value 1 in an accepted input vector. The modified circuit $C^{\prime}$ accepts a weight $k+1$ input vector if and only if $C$ accepts a weight $k$ input vector. For weft at least two, the transformation is weft-preserving and yields a circuit $C^{\prime}$ with bottommost And gate, but possibly with not gates at the lower levels. Thus it may be necessary to repeat Steps 2 and 3 to obtain a homogenized circuit with bottommost And gate.

Step 5. Organizing the small gates.

The tree circuit $C$ received from the previous step has the following properties: (i) the output gate is an And gate, (ii) from the bottom, the circuit consists of layers which alternately consist of only And gates or only $O r$ gates, for up to $t$ layers, and (iii) above this, there are branches $B$ of height $h^{\prime}=h-t$ consisting only of small gates. Since a small gate branch $B$ has bounded depth, it has at most $2^{h^{\prime}}$ gates, and thus in constant time (since $h$ is fixed), we can find either (1) an equivalent sum-of-products circuit with which to replace $B$, as required 
by Case 1 of Step 6 below, or (2) an equivalent product-of-sums circuit, as required by Case 2 of Step 6.

In this step, all such small gate branches $B$ of $C$ are replaced in this way, appropriately for the relevant case of Step 6. In Case 1, the depth 2 sum-of-products circuits replacing the small gate branches $B$ have a bottomost or gate $g_{B}$ of fan-in at most $2^{2^{h^{\prime}}}$, and the and gates feeding into $g_{B}$ have fan-in at most $2^{h^{\prime}}$, so the weft of the circuit has been preserved by this transformation, which may increase the size of $C$ by the constant factor $2^{2^{h^{\prime}}}$. The topmost level of large gates (to which the branches $B$ are attached in $C$ ) consists of $O r$ gates, in Case 1, so that the gates $g_{B}$ can be merged into this topmost level. Merging is performed similarly in Case 2, where the replacing circuits are products-of-sums, and the topmost level of large gates consists of And gates. For the next step we consider two cases, depending on whether the topmost level of large gates consists of $O r$ gates or And gates. (Essentially, this corresponds to whether weft $t$ is even or odd.)

Step 6. A monotone change of variables (two cases).

In this step (in both cases) we employ a "change of variables" based on the combinatorial reduction of Theorem 2.1. The goal is to obtain an equivalent circuit that has the property that either all the inputs are MONOTONE (Case 1) (i.e., no inverters in the circuit), or all the inputs are negated with no other inverters in the circuit, which we call ANTIMONOTONE. (Actually in this case we will have some of the inputs positive but these will only be enforcers as we will see. So we should call this case NEARLY ANTIMONOTONE) (Case 2). The point of this step becomes apparent in the next step when we use the special character of the circuit thus constructed to enable us to eliminate the small gates.

Consider the reduction of Theorem 2.1, especially in the light of the remark following the proof. This reduction consists of two parts. The first is the ring of selection gadgets which allow variable choice, gap choice, and then gap enforcement; the second part is consistency obtained by clause wiring. The idea is to "hard wire" the selection and consistency parts of the construction into the circuit, the point being that we can replace positive instances of variable fan-out in the original circuit by outputs corresponding to choice of that variable in the positive selection component. We can replace negative fan-out in the original circuit by the appropriate sets of gap variables. Finally we can wire in the fact that we need a dominating set and other enforcements by using the facts that we will only look at a weight $2 k$ input, and an And of $O r$ 's, which will not add to the weft of the circuit. We argue more precisely below, and also prove in two parts that the whole process $\mathrm{cn}$ be accomplished without increasing weft, given that the weft is $\geq 2$.

Suppose the inputs to the circuit $C$ received at the beginning of this step are $x[1], \ldots, x[n]$, and suppose that the output gate of $C$ is an And gate. Let $Y$ denote the boolean expression having $2 n$ clauses, with each clause consisting of a single literal, and with one clause for each of the $2 n$ literals of the $n$ input variables. The reduction of Theorem 2.1 allows us to translate $Y$ into a monotone formula via dominating set, thus capturing monotonically all the relevant input settings. Thus, let $G_{Y}$ be the graph constructed for this expression as in the proof of Theorem 2.1. Note that only part of $G_{Y}$ will actually be wired into $C$.

Keeping this in mind, and using the variable (vertex) set obtained from $G_{Y}$, the change of variables is implemented for $C$ as follows. (1) Create a new input for each vertex of $G_{Y}$ that is not a clause vertex. (2) Replace each positive input fan-out of $x[i]$ in $C$ with an $O r$ gate having $k$ new input variable arguments corresponding to the vertices to which the clause vertex for the clause $(x[i])$ of $Y$ is adjacent in $G_{Y}$. (3) Replace each negated fan-out line of $x$ [i] with an $O r$ gate having $O\left(n^{2}\right)$ new input variable arguments corresponding to the vertices to which the clause vertex for the clause $(\neg x[i])$ of $Y$ is adjacent in $G_{Y}$. (4) Merge with the output $A$ nd gate of $C$ a new circuit branch corresponding to the product-of-sums expression, 


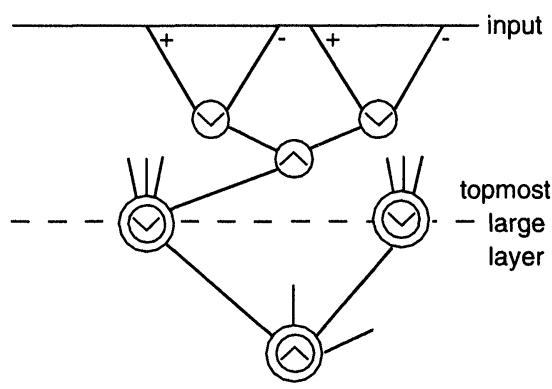

(a)

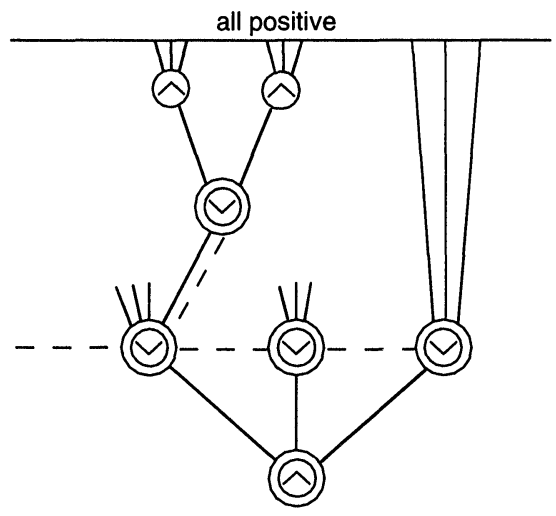

(c)

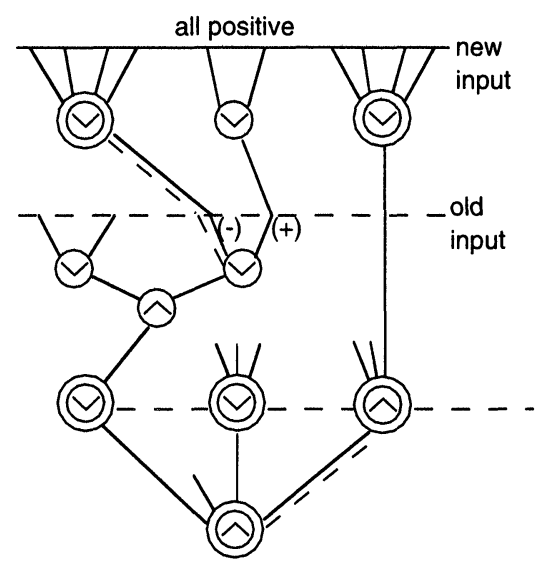

(b)

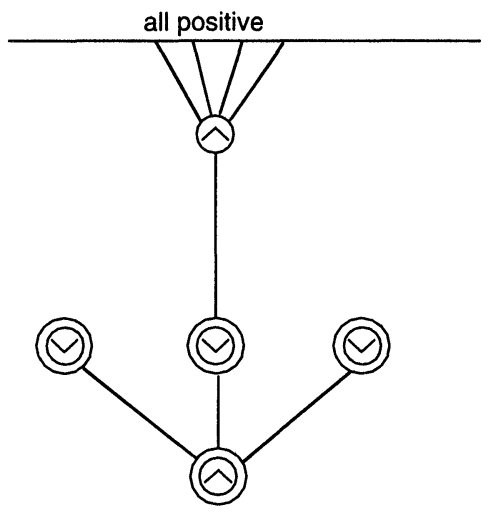

(d)

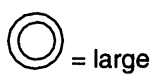

$O=$ small

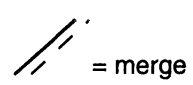

FIG. 2. A topmost layer of large Or gates.

where the product is taken over all nonclause vertices of $G_{Y}$, and the sum for a vertex $u$ is the sum of the new inputs corresponding to the nonclause vertices in $N[u]$ (this is the dominating set and other enforcements).

The modified circuit $C^{\prime}$ obtained in this way accepts a weight $2 k$ input vector if and only if the original circuit $C$ accepts a weight $k$ input vector. The proof of this is essentially the same as for Theorem 2.1. If all of the not gates of $C$ are at the top, then the circuit $C^{\prime}$ will be MONOTONE. However, to see that this change of variables can be employed to obtain a monotone or nearly antimonotone circuit without increasing weft, we must consider two cases.

Case 1. The topmost large-gate level consists of Or gates.

Let $C$ denote the circuit obtained from Step 5 and perform a change of variables as described above. The sequence of transformations of $C$ for this step is shown schematically in Fig. 2. 
The result is a circuit $C^{\prime}$ with no not gates. The input weight we are now concerned with is $2 k$, and the construction of $C^{\prime}$ from $C$ may involve quadratic blow-up.

Next, we move the small and gates on the second level upward past the $O r$ gates introduced by the change of variables, and then merge the $O r$ gates down to the topmost large layer (of Or gates).

Case 2. The topmost large-gate level consists of And gates.

Here we use a similar argument, beginning with a trick. Below each gate of the topmost large-gate level (of And gates), a double negation is introduced (equivalently). One of the not gates is moved to the top of the circuit (by DeMorgan's identities). This is followed by a change of variables based on Theorem 2.1, as in Case 1. The second level and gates are commuted upwards, and the $O r$ gates of the second and third levels are merged, as in Case 1. So now the circuit has no negated inputs and no inverters except the residual ones below the top layer of $O r$ gates. Finally, these remaining not gates are commuted to the top. Note that this means that all fanouts are negated except the ones to the enforcement $O r$ gate added during Step 4.

We are now in position for the last step.

Step 7. Eliminating the remaining small gates.

If we regard the inputs to $C$ as variables, this step consists of another "change of variables." Let $k$ be the relevant weight parameter value supplied by the last transformation. In this step we will produce a circuit $C^{\prime}$ corresponding directly to a $t$-normalized boolean expression (that is, consisting only of $t$ alternating layers of $A n d$ and $O r$ gates) such that $C$ accepts a weight $k$ input vector if and only if $C^{\prime}$ accepts a vector of weight $k^{\prime}=k \cdot 2^{k+1}+2^{k}$.

Suppose that $C$ has $m$ remaining small gates. In Case 1, these are and gates and the inputs are all positive. In Case 2 , these are or gates and the inputs to these gates are all negated. For $i=1, \ldots, m$ we define the sets $A_{i}$ of the inputs to $C$ to be the sets of input variables to these small gates. The central idea for this step is to create new inputs representing the sets $A_{i}$ of inputs to $C$.

For example, suppose (Case 1) that the output of the small and gate $g_{i}$ in $C$ is the boolean product $(a b c d)$ of the inputs $a, b, c, d$ to $C$. Thus $A_{i}=\{a, b, c, d\}$. The gate $g_{i}$ can be eliminated by replacing it with an input line from a new variable $v[i]$ which represents the predicate $a=b=c=d=1$. (This representation, of course, will need to be enforced by additional circuit structure.) Similarly (Case 2$)$ if $g_{i}$ computes the value $(\bar{a}+\bar{b}+\bar{c}+\bar{d})$ then $g_{i}$ can be replaced by a negated input line from $v[i]$.

Let $x[j]$ for $j=1, \ldots, s$ be the input variables to $C$. We introduce new input variables of the following kinds:

(1) One new variable $v[i]$ for each set $A_{i}$ for $i=1, \ldots, m$ to be used as indicated above.

(2) For each $x[j]$ we introduce $2^{k+1}$ copies $x[j, 0], x[j, 1], x[j, 2], \ldots, x\left[j, 2^{k+1}-1\right]$.

(3) "Padding" consisting of $2^{k}$ meaningless variables (inputs not supplying output to any gates) $z[1], \ldots, z\left[2^{k}\right]$.

We add to the circuit an enforcement mechanism for the change of variables. The necessary requirements can be easily expressed in P-o-S form, and thus can be incorporated into the bottom two levels of the circuit as additional Or gates attached to the bottommost (output) And gate of the circuit.

We require the following implications concerning the new variables:

(1) The $s \cdot 2^{k+1}$ implications, for $j=1, \ldots, s$ and $r=0, \ldots, 2^{k+1}-1$,

$$
x[j, r] \Rightarrow x\left[j, r+1 \quad\left(\bmod 2^{k+1}\right)\right] .
$$

(2) For each containment $A_{i} \subseteq A_{i^{\prime}}$, the implication

$$
v\left[i^{\prime}\right] \Rightarrow v[i]
$$


(3) For each membership $x[j] \in A_{i}$, the implication

$$
v[i] \Rightarrow x[j, 0] \text {. }
$$

(4) For $i=1, \ldots, m$ the implication

$$
\left(\prod_{x[j] \in A_{i}} x[j, 0]\right) \Rightarrow v[i] .
$$

It may be seen that this transformation increases the size of the circuit by a linear factor exponential in $k$. We make the following argument for the correctness of the transformation.

If $C$ accepts a weight $k$ input vector, then setting the corresponding copies $x[i, j]$ among the new input variables accordingly, together with appropriate settings for the new "collective" variables $v[i]$ yields a vector of weight between $k \cdot 2^{k+1}$ and $k \cdot 2^{k+1}+2^{k}$ that is accepted by $C^{\prime}$. The reason the weight of this corresponding vector may fall short of $k^{\prime}=k \cdot 2^{k+1}+2^{k}$ is that not all of the subsets of the $k$ input variables to $C$ having value 1 may occur among the sets $A_{i}$. An accepted vector of weight exactly $k^{\prime}$ can be obtained by employing some of the "padding" input variables $z[i]$ to $C^{\prime}$

Note that the seemingly simpler strategy of creating a new input variable for each set of at most $k$ inputs to $C$ would not serve our purposes, since it would involve increasing the size $n$ of the circuit to possibly $n^{k}$. (We are limited in our computational resources for the reduction to $f(k) n^{\alpha}$. The constant $\alpha$ can be an arbitrary function of the depth and weft bounds $h$ and $t$, but not $k$.)

For the other direction, suppose $C^{\prime}$ accepts a vector of weight $k^{\prime}$. Because of the implications in (1) above, exactly $k$ sets of copies of inputs to $C$ must have value 1 in the accepted input vector. Because of implications (2)-(4), the variables $v[i]$ must have values in the accepted input vector compatible with the values of the sets of copies. By the construction of $C^{\prime}$, this implies there is a weight $k$ input vector accepted by $C$.

COROLLARY 3.8. (i) For $t>0$, MONOTONE $W[2 t]=W[2 t]$.

(ii) For $t>0$ WEIGHTED MONOTONE $2 t$-NORMALIZED SATISFIABILITY is $W[2 t]$ complete.

(iii) For $t>0$, MONOTONE $2 t+1$-NORMALIZED SATISFIABILITY is in W[2t].

Proof. (i) The proof come from the analysis of Step 6, Case 1.

(ii) After Step 7, we can apply Step 6, Case 1 (again) to a $2 t$-normalized formula. The result is a $2 t$ normalized monotone formula. (This time all small gates go away by gluing the DOMINATING SET reduction, or Theorem 2.1.)

(iii) This result follows by the transformations of Step 7, applied to the a $2 t+1$-normalized monotone formula.

We remark that Corollary 3.8 leads one to conjecture that MONOTONE $W[2 t+1]=$ $W[2 t]$.

We also remark that in Downey and Fellows [56] we have proven the complementary result for $W[2 t+1]$ by showing that $W[2 t+1]$ contains ANTIMONOTONE $2 t+2$-NORMALIZED SATISFIABILITY. It is an open question whether ANTIMONOTONE CNFSAT is in $W[1]$. The problem is that the relevant gadgets seem to need two levels to enact. Note that the above theorem fails to identify a problem complete for $W[1]$. In [56] we will also show that INDEPENDENT SET and a number of other natural parameterized problems are complete for $W[1]$. There we show that $W[1]=W[1,2]$ where $W[1,2]$ is equivalent to the problem of, given a formula $X$ in conjunctive normal form and of clause size two, does $X$ have a satisfying assignment of weight $k$ ? While the unparameterized problem that considers $(X, k)$ is $N P$-complete (easy reduction from INDEPENDENT SET), variations of this are classically 
$P$-time or $F P T$. For instance the problem that asks if there is any satisfying assignment is well known to be $P$-time, and the problem that asks if there is a satisfying assignment of weight less than or equal to $k$ is $F P T$ as follows. Let $X$ be the $2-C N F$ formula and let $k$ be given. If $X$ has no clause without negated literals, we are done since the assignment with all false works. Otherwise choose some clause $C$ with only positive literals. One of these must be made true so we begin a tree of possibilities. Continue inductively in this way. As the clause size is bounded here by 2 this only gives a factor of $2^{k}$.

The $W[t]$ hierarchy reflects, in a finely resolved way, the difficulty of "solution checking." What happens if, more bluntly, we simply address fixed-parameter complexity for problems for which solutions can be checked in polynomial time? To study this question, as we mentioned earlier, it is natural to define the following complexity classes.

DEFINITION 3.9. A parameterized problem L belongs to $W[P](W[S A T])(M O N O T O N E$ $W[P])$ if $L$ uniformly reduces to the parameterized circuit problem $L_{\mathcal{F}}$ for some family of (Boolean) (monotone) circuits $\mathcal{F}$.

Note that $W[t]$ is contained in $W[P]$ for every $t$, and that $W[P]=F P T$ if $P=N P$. With Karl Abrahamson, [1], [2], we have been able to show that all of the problems identified in Abrahamson et al. [3] as complete for $P G T$ are uniformly complete for $W[P]$. (For the reasons mentioned before, we would argue that the present theory offers a better framework for those results and allows us to address much wider parameterized issues.) We have also identified a number of further natural complete problems. These results and some aspects of the structure of $W[P]$ as well as parameterized $P S P A C E$ are reported in Abrahamson, Downey, and Fellows [1], [2]. For PSPACE there are very interesting problems that seem hard and are natural ones that relate to winning strategies for $k$-move games.

4. Summary and open problems. We have presented in this paper a basic framework and fundamental completeness results for the study of fixed-parameter tractability. We view the exploration of this topic as a large project, of which this constitutes only the initial step. As can be seen from the appendix and the reference list, in the time that this paper has been in the refereeing/publication process, there has already been quite a bit of work using our classification. We believe that our techniques are of particular interest in the area of molecular biology, and because of the fact that although problems may have no polynomial time approximation scheme unless $P=N P$, they can certainly still be in $F P T$.

In some ways, the study of fixed-parameter tractability and completeness addresses the subject of computational infeasibility inside of $P$. For related work from a different perspective see Buss and Goldsmith [35] and the references cited there. Many of the approaches and issues concerning the standard complexity classes have natural analogues in this setting that are thus far unexplored.

Consider, for example, the issue of parallel complexity. Trivially, there is a parallel algorithm running in time $O(\log n)$ and using $n^{k}$ processors to determine if a graph $G$ on $n$ vertices has a dominating set of size $k$, for each fixed $k$. For a contrasting result, Lagergren [95] has shown that for each fixed $k$, it can be determined in time $O\left(\log ^{3} n\right)$ with $O(n)$ processors whether a graph has treewidth at most $k$. This suggests a natural fixed-parameter analogue of $N C$. Similar remarks apply to randomized complexity. With Ken Regan, the authors have made a little progress with randomized complexity [64].

For another example, consider approximation algorithms. One of the fundamental results of Robertson and Seymour (apart from their work on graph minors) is that there is an algorithm that in time $f(k) \cdot n^{2}$ finds, for a graph $G$ of order $n$, either (1) a tree decomposition of width at most $5 k$, or (2) evidence that the treeewidth of $G$ is greater than $k$. (Of course this is now replaced by Bodlaender's linear time algorithm [23].) An analogous result for DOMINATING SET might be an algorithm running in time $f(k) \cdot n^{c}$ that finds either (1) a dominating set 
of size $O(k)$, or (2) evidence that the minimum size of a dominating set for $G$ is greater than $k$. Such an algorithm is presently unknown. It may even be that the existence of such an algorithm would imply the collapse of the $W$-Hierarchy, much as the existence of a $P$ time relative approximation algorithm for the TRAVELING SALESPERSON problem would imply $P=N P$ (Garey and Johnson [81]).

Many interesting structural questions concerning the $W$-Hierarchy remain to be explored. For instance we have established some connections between classical classes and our parameterized classes. Thus if $W[2 t] \subseteq F P T$, then $2 t$-NORMALIZED SATISFIABILITY is soluable in time $p(n) 2^{o(v)}$, where $n$ is the size of the input, $p$ is a polynomial, and $v$ is the number of variables. For this result and other structural results along these lines, see Abrahamson, Downey, and Fellows [2]. Nevertheless, the precise relationship between classical classes and the parameterized one is still unclear. We also are not aware of an oracle separating the $W$-Hierarchy, nor do we know if collapse propogates upwards. (That is, if $F P T=W[t]$ implies $F P T=W[t+1]$, for instance.)

From a concrete point of view, we also do not know if a problem such as the following belongs to $W[t]$ for any $t$.

\section{TWO PLAYER DOMINATING SET}

Instance: A graph $G=(V, E)$ and a positive integer $k$.

Question: Is it true that for every $k$-element subset $V^{\prime} \subseteq V$, there is a $k$-element subset $V^{\prime \prime} \subseteq V$ such that $V^{\prime} \cup V^{\prime \prime}$ is a $2 k$-element dominating set for $G$ ?

We have been able to prove the following density theorem.

THEOREM 4.1. For the strong uniform reduction hierarchy, if any of the containments

$$
F P T \subseteq W[1] \subseteq W[2] \subseteq \cdots
$$

is proper, then there are infinitely many intervening equivalence classes of parameterized problems with respect to strong uniform reductions.

Actually, we can prove a much stronger result along the lines of the full Ladner [94] theorem. Also Downey and Fellows [58] contains quite a number of other structural and relativization results. For instance we know that there is an oracle with $P \neq N P$ yet the $W$ Hierarchy collapses. It is an open question whether an analogue of Theorem 4.1 holds in the uniform case. We remark that the setting of parameterized problems introduces some technical challenges for density results. Our proof of Theorem 4.1 [58] employs techniques from the infinite-injury priority method. Technically while the standard polynomial time reductions are $\Sigma_{2}$ (on recursive languages), the fact is that strong uniform reducibility is $\Sigma_{3}$-complete, and the other two reductions are $\Sigma_{4}$-complete [61]. (The last result needs a tree of strategies infinite injury priority argument.)

Finally, we think the primary value of our theory of fixed-parameter tractability is that there is, for many parameterized problems, a compelling practical interest. There are many natural parameterized problems that may well be complete for various levels of the $W$-Hierarchy. Demonstrations of such completeness would provide an explanation of why, although they are soluable in polynomial time for each fixed parameter value, these problems resist attempts to show fixed-parameter tractability.

5. Appendix: A problem compendium and guide to W-Hierarchy completeness, hardness, and classification, and some open questions. This appendix contains problem definitions and summaries of most of the presently known completeness and hardness results, and information concerning fixed-parameter tractability for restrictions of problem instances. References are given where appropriate. The problems discussed are grouped (more or less) 
according to level in the $W$-Hierarchy. Our list for $F P T$ is obviously incomplete, but the given examples should provide the reader with the flavour of such results.

\subsection{In FPT.}

Remark. The following is only a small list of the known FPT problems. Many more can be obtained by Courcelle's theorem applied to classes of bounded tree width, in conjunction with Bodlaender's theorem on Treewidth $k$ recognition. (See e.g., Abrahamson and Fellows [4], [5], Courcelle [49], Downey and Fellows [61], or Van Leeuwen [115].)

\section{CROSSING NUMBER FOR MAX DEGREE 3 GRAPHS}

Instance: A graph $G$ all of whose vertices have max degree 3 .

Parameter: A positive integer $k$.

Question: Does $G$ have a an embedding with crossing number $\leq k$ ?

This is $O\left(n^{3}\right)$ by [69] via [106], [107].

\section{ALTERNATING HITTING SET}

Instance: A collection $C$ of subsets of a set $B$ with $|c| \leq k_{1}$ for all $c \in C$, an integer $k_{2}$.

Parameter: A positive integer $k k_{1}, k_{2}$.

Question: Does player one have a forced win in $\leq k_{2}$ moves in the following game played on $C$ and $B$ ? Players alternate choosing a new element of $B$ until, for each $c \in C$, some member of $c$ has been chosen. The player whose choice causes this to happen loses.

The general version of this problem is $P S P A C E$-complete by a reduction from $Q B F$ (see Garey and Johnson [81, Bp7]). This problem is in FPT by Abrahamson, Downey, and Fellows [2]. Soluable in $O(n)$ time for fixed $k_{1}$ and $k_{2}$.

\section{CUTWIDTH}

Instance: A graph $G=(V, E)$.

Parameter: A positive integer $k$.

Question: Is the cutwidth of $G \leq k$ ?

The general version of this problem is $N P$-complete by a reduction from SIMPLE MAX CUT (see Garey and Johnson [81, GT44]). This problem is in FPT by Fellows and Langston [75]. Soluable in $O(n)$ time for fixed $k$ (Bodlaender [17]).

\section{DIAMETER IMPROVEMENT FOR PLANAR GRAPHS}

Instance: A planar graph $G=(V, E)$.

Parameter: A positive integer $k$.

Question: Can $G$ be augmented with additional edges in such a way that the resulting graph $G^{\prime}$ remains planar and the diameter of $G^{\prime}$ is at most $k$ ?

This problem is in FPT by Downey and Fellows [59] after Robertson and Seymour [106]. Soluable in $O(n)$ time for fixed $k$ (Bodlaender [17]).

MINIMUM FILL-IN

Input: A graph $G$.

Parameter: A positive integer $k$.

Question: Can we add $\leq k$ edges to $G$ and cause $G$ to become chordal?

The general problem is $N P$ complete by Yannakakis [119]. Soluable in time $O\left(c^{k} .|E|\right)$ and $O\left(k^{5}|E||V|+f(k)\right)$ by Kaplan, Shamir, and Tarjan [91].

\section{DISJOINT PATHS}

Instance: A graph $G=(V, E), s_{1}, \ldots, s_{k} \in V$ start vertices, $t_{1}, \ldots, t_{k} \in V$ end vertices. 
Parameter: $k$

Question: Do there exist vertex disjoint paths $P_{1}, \ldots, P_{k}$ such that $P_{i}$ starts at vertex $s_{i}$ and ends at vertex $t_{i}$ for $i=1 \ldots k$ ?

The general version of this problem is NP-complete by a reduction from 3SAT (see Garey and Johnson [81, ND40]). This problem is in F PT by Robertson and Seymour [106]. Soluable in $O\left(n^{3}\right)$ time for fixed $k$.

\section{FEEDBACK VERTEX SET}

Instance: A graph $G=(V, E)$.

Parameter: A positive integer $k$.

Question: Is there a set $U$ of $k$ vertices of $G$ such that each cycle of $G$ passes through some vertex of $U$ ?

The general version of this problem is NP-complete by a reduction from VERTEX COVER (see Garey and Johnson [81, GT7]). This problem is in FPT by Downey and Fellows [59] and Bodlaender [16]. Soluable in $O(n)$ time for fixed $k$.

\section{GATE MATRIX LAYOUT}

Instance: A boolean matrix $M$.

Parameter: A positive integer $k$.

Question: Is there a permutation of the columns of $M$ so that, if in each row we change to * every 0 lying between the row's leftmost and rightmost 1 , then no column contains more than $k 1$ 's and *'s?

This problem is in FPT by Fellows and Langston [77]. Soluable in $O(n)$ time for fixed $k$ Bodlaender [17]. Equivalent to GRAPH PATHWIDTH.

\section{GRAPH GENUS}

Instance: A graph $G=(V, E)$.

Parameter: A positive integer $k$.

Question: Does $G$ have genus $k$ ?

The general version of this problem is $N P$-complete. This problem is in FPT by Fellows and Langston [74] via Robertson and Seymour [106]. Soluable in time $O\left(n^{3}\right)$ for fixed $k$ by the results of Robertson and Seymour.

\section{LONG CYCLE}

Instance: A graph $G=(V, E)$.

Parameter: A positive integer $k$.

Question: Does $G$ have a cycle of length $\geq k$ ?

The general version of this problem is $N P$-complete by a reduction from HAMILTON CIRCUIT (see Garey and Johnson [81, ND28]). This problem is in FPT by Fellows and Langston [74]. Soluable in $O(n)$ time for fixed $k$.

\section{MAX LEAF SPANNING TREE}

Instance: A graph $G=(V, E)$.

Parameter: A positive integer $k$.

Question: Does $G$ have a spanning tree with $k$ or more leaves?

The general version of this problem is $N P$-complete by a reduction from DOMINATING SET (see Garey and Johnson [81, ND2]). This problem is in FPT by Downey, and Fellows [59] and Bodlaender [16]. In LOGSPACE + Advice by Cai et al. [41]. Soluable in $O(n)$ for fixed $k$. 


\section{MINIMUM DISJUNCTIVE NORMAL FORM}

Instance: A set $X=\left\{x_{1}, x_{2}, \ldots, x_{n}\right\}$ of variables, a set $A \subseteq\{0,1\}^{n}$ of implicants, a positive interger $m$.

Parameter: $|A|$.

Question: Is there a DNF expression $E$ over $X$, having no more than $m$ disjuncts, such that $E$ is true for precisely those truth assignments in $A$ and no others?

The general version of this problem is $N P$-complete by a reduction from SET COVER (see Garey and Johnson [81, LO9]). This problem is in FPT by [66]. Soluable in $O\left(2^{|A|} n\right.$ ) time for fixed $A$.

\section{MINOR ORDER TEST}

Instance: Graphs $G=(V, E)$ and $H=\left(V^{\prime}, E^{\prime}\right)$.

Parameter: $H$

Question: Is $H \leq_{\text {minor }} G$ ?

The general version of this problem is $N P$-complete by a reduction from HAMILTON CIRCUIT (see Garey and Johnson [81, OPEN2]) This problem is in FPT by Robertson and Seymour [106]. Soluable in $O\left(n^{3}\right)$ time for fixed $k$.

\section{PLANAR FACE COVER}

Instance: A graph $G=(V, E)$.

Parameter: A positive integer $k$.

Question: Can $G$ be embedded in the plane so that there are $k$ faces which cover all vertices?

The general version of this problem is $N P$-complete by a reduction from VERTEX COVER (Fellows [70]). This problem is in FPT by Bienstock and Monma [15]. Soluable in $O(n)$ time for fixed $k$.

\section{SEARCH NUMBER}

Instance: A graph $G=(V, E)$.

Parameter: A positive integer $k$.

Question: Are $k$ searchers sufficient to ensure the capture of a fugitive who is free to move with arbitrary speed about the edges of $G$ ?

The general version of this problem is NP-complete. This problem is in FPT by Fellows and Langston [75]. Soluable in $O(n)$ time for fixed $k$ (Bodlaender [17]).

\section{STEINER TREE}

Instance: A graph $G=(V, E)$, a set $S$ of at most $k$ vertices in $V$, an integer $m$.

Parameter: $k$

Question: Is there a set of vertices $T \subseteq V-S$ such that $|T| \leq m$ and $G[S \cup T]$ is connected?

The general version of this problem is $N P$-complete by a reduction from EXACT COVER (see Garey and Johnson [81, ND12]) [82]. This problem is in FPT by Dreyfus and Wagner [65]. Soluable in time $O\left(3^{k} n+2^{k} n^{2}+n^{3}\right)$ by the Dreyfus-Wagner algorithm (Wareham [117]).

\section{TREEWIDTH}

Instance: A graph $G=(V, E)$.

Parameter: A positive integer $k$.

Question: Does $G$ have treewidth $k$ ?

The general version of this problem is $N P$-complete [8]. Soluable in $O(n)$ time for fixed $k$ (Bodlaender [23]). 


\section{VERTEX COVER}

Instance: A graph $G=(V, E)$.

Parameter: A positive integer $k$.

Question: Does $G$ have a vertex cover of size $\leq k$ ?

The general version of this problem is NP-complete by a reduction from 3SAT (see Garey and Johnson [81, GT1]). This problem is in FPT by Downey and Fellows [59] and Buss [34]; and in fact in LOGSPACE + Advice by Cai et al. [41]. Soluable in $O\left(2^{k} n\right)$ or $O\left(n+k^{k}\right)$ time.

\section{$k$-PERFECT MATCHINGS}

Input: A graph $G$.

Parameter: A positive integer $k$.

Question: Does $G$ have at least (or exactly) $k$ perfect matchings?

The general problem of finding the maximum number of perfect matchings is \# $P$ complete (in the size of $G$ ) even for bipartite graphs by Valiant [113]. For $k=1$, the problem is in $P$ for bipartite graphs by the old work of Ford and Fulkerson, and for general graphs by the work of Edmonds. The problem is $O(k . e)$, where $e$ denotes the number of edges, for any fixed $k$ by Itai, Rodeh, and Tanimoto [89]. For weighted graphs one finding the best $k$ matchings is FPT by, for instance, Chegireddy and Hamacher [46].

\section{SHORT 3DIMENSIONAL MATCHING}

Input: A graph $G \subseteq X \times Y \times Z$ with $|X|=|Y|=|Z|$.

Parameter: A positive integer $k$.

Question: Does there exist a subset $G^{\prime} \subseteq G$ such that $\left|G^{\prime}\right|=k$ and for all $\langle x, y, z\rangle \neq\left\langle x^{\prime}, y^{\prime}, z^{\prime}\right.$ both in $G^{\prime}$, we have $x \neq x^{\prime}, y \neq y^{\prime}$, and $z \neq z^{\prime}$ ?

The general problem with $k$ varying is $N P$ complete and is one of Garey and Johnson's six basic problems. For $k$ fixed the problem is $F P T$ by Downey and Fellows [61].

\subsection{In FPT(nonuniform).}

\section{GRAPH LINKING NUMBER}

Instance: A graph $G=(V, E)$.

Parameter: A positive integer $k$.

Question: Can $G$ be embedded into 3-space such that the maximum size of a collection of topologically linked disjoint cycles is bounded by $k$ ?

This problem is in FPT(nonuniform) by Fellows and Langston [74] after Robertson and Seymour [106]. Soluable in $O\left(n^{3}\right)$ time for fixed $k$.

\subsection{In randomized FPT.}

\section{BOUNDED FACTOR FACTORIZATION}

Instance: An $n$-bit positive integer $N$.

Parameter: A positive integer $k$.

Question: Is there a prime factor $p$ of $N$ such that $p<n^{k}$ ?

This problem is in randomized FPT by Fellows and Koblitz [72], [73].

\section{LINEAR EXTENSION COUNT}

Instance: A poset $(P, \leq)$.

Parameter: A positive integer $k$.

Question: Does $P$ have at least $k$ linear extensions? 
This problem is in randomized FPT by Brightwell and Winkler [32], [33]. Not known to be in FPT.

\section{POLYNOMIALLY SMOOTH NUMBER}

Instance: An $n$-bit positive integer $N$.

Parameter: A positive integer $k$.

Question: Is $N n^{k}$-smooth, i.e., is every prime divisor of $N$ bounded by $n^{k}$ ?

This problem is in randomized FPT by Fellows and Koblitz [72], [73]. $n^{k}$-smoothness of $n$-digit numbers is a natural number-theoretic property that arises in the study of polynomialtime complexity. For example, the concept plays a central role in the demonstration that primality is in $U P \cap$ co-UP.

\section{SMALL PRIME DIVISOR}

Instance: An $n$-bit positive integer $N$.

Parameter: A positive integer $k$.

Question: Does $N$ have a nontrivial divisor less than $n^{k}$ ?

This problem is in randomized FPT by Fellows and Koblitz [72], [73].

\section{4. $W[1]-$ complete.}

\section{$t$ THRESHOLD STABLE SET}

Instance: A directed graph $G=(V, A)$.

Parameter: A positive integer $k$.

Question: Does $G$ have a stable set of size $k$ ? (A stable set is a set of vertices $V^{\prime} \subseteq V$ such that for every vertex $v$ of $V-V^{\prime}$, there are fewer than $t$ vertices $u \in V^{\prime}$ with $u v \in A$.)

This problem is $W[1]$-complete by a reduction from INDEPENDENT SET. Complete for $W[1]$ by Downey and Fellows [61].

\section{BINARY CLADISTIC CHARACTER COMPATIBILITY}

Instance: A set $C$ of $n$ binary cladistic characters over $m$ objects.

Parameter: A positive integer $k$.

Question: Is there a subset $C^{\prime} \subseteq C,\left|C^{\prime}\right|=k$, such that all pairs of characters in $C^{\prime}$ are compatible?

The general version of this problem is $N P$-complete by a reduction from CLIQUE (Day and Sankoff [52]). This problem is $W[1]$-complete by the same reduction (Wareham [117]). The unconstrained-character version of this problem is also W[1]-complete (Wareham [117]). If $k=|C|$, one obtains the problem TRIANGULATING COLORED GRAPHS (PERFECT PHYLOGENY).

\section{BINARY QUALITATIVE CHARACTER COMPATIBILITY}

Instance: A set $C$ of $n$ binary qualitative characters over $m$ objects.

Parameter: A positive integer $k$.

Question: Is there a subset $C^{\prime} \subseteq C,\left|C^{\prime}\right|=k$, such that all pairs of characters in $C^{\prime}$ are compatible?

The general version of this problem is $N P$-complete by a reduction from BINARY CLADISTIC CHARACTER COMPATIBILITY (Day and Sankoff [52], Wareham [117]). This problem is $W[1]$-complete by the same reduction. The unconstrained-character version of this problem is $W[1]$-hard (Wareham [117]). 


\section{CLIQUE}

Instance: A graph $G=(V, E)$, a positive integer $k$.

Parameter: A positive integer $k$.

Question: Is there a set of $k$ vertices $V^{\prime} \subseteq V$ that forms a complete subgraph of $G$ (that is, a clique of size $k$ )?

The general version of this problem is $N P$-complete by a reduction from VERTEX COVER (see Garey and Johnson [81, GT19]). This problem is $W[1]$-complete by a reduction from INDEPENDENT SET by Downey and Fellows [56]. Fixed-parameter tractable for planar graphs and for graphs of maximum degree $f(k)$ for any fixed function $f$.

\section{INDEPENDENT SET}

Instance: A graph $G=(V, E)$, a positive integer $k$.

Parameter: A positive integer $k$.

Question: Is there a set $V^{\prime} \subseteq V$ of cardinality $k$, such that $\forall u, v \in V^{\prime}, u v \notin E$ ?

The general version of this problem is $N P$-complete by a reduction from VERTEX COVER (see Garey and Johnson [81, GT20]). This problem is $W[1]$-complete by a reduction from ANTIMONOTONE $W[1,2]$ by Downey and Fellows [56]. Fixed-parameter tractable for planar graphs.

\section{LONGEST COMMON SUBSEQUENCE (I)}

Instance: A set of $k$ strings $X_{1}, \ldots, X_{k}$ over an alphabet $\Sigma$, a positive integer $m$.

Parameter: $k, m$

Question: Is there a string $X \in \Sigma^{*}$ of length at least $m$ that is a subsequence of $X_{i}$ for $i=1, \ldots, k$ ?

The general version of this problem is $N P$-complete by a reduction from VERTEX COVER (see Garey and Johnson [81, SR10]). This problem is $W[1]$-complete by a reduction from CLIQUE by Bodlaender et al. [25].

\section{$d$-RED/BLUE NONBLOCKER}

Instance: A graph $G=(V, E)$ of maximum degree $d$ where $V$ is partitioned into two color classes $V=V_{\text {red }} \cup V_{\text {blue }}$.

Parameter: A positive integer $k$.

Question: Is there is a set of red vertices $V^{\prime} \subseteq V_{\text {red }}$ of cardinality $k$ such that every blue vertex has at least one neighbor that does not belong to $V^{\prime}$ ?

This problem is $W[1]$-complete by a reduction from $W[1, s]$ (Downey and Fellows [56]).

\section{SEMIGROUP EMBEDDING}

Instance: A semigroup $(S, \cdot)$.

Parameter: A positive integer $k$ and a semigroup $(H, x)$.

Question: Can $H$ be embedded into $S$ ?

This problem is $W[1]$-complete by a reduction from CLIQUE (Downey and Fellows [61]).

\section{SEMILATTICE EMBEDDING}

Instance: A semilattice $L$.

Parameter: A positive integer $k$ and a semilattice $H$.

Question: Is $H$ embeddable into $L$ ?

This problem is $W[1]$-complete by a reduction from CLIQUE (Downey and Fellows [61]). 


\section{SET PACKING}

Instance: A finite family of sets $S=S_{1}, \ldots, S_{n}$, an integer $k$.

Parameter: $k$

Question: Does $S$ contain a subset $R$ of $k$ mutually disjoint sets?

The general version of this problem is $N P$-complete by a reduction from $\mathrm{X} 3 \mathrm{C}$ (see Garey and Johnson [81, SP3]). This problem is $W[1]$-complete by a reduction from INDEPENDENT SET (Ausiello, D'Atri, and Protasi [11], Wareham [117]).

\section{SHORT CONTEXT SENSITIVE DERIVATION}

Instance: A context-sensitive grammar $G=(N, \Sigma, \Pi, S)$, a word $x \in \Sigma^{*}$.

Parameter: A positive integer $k$.

Question: Is there a $G$-derivation of $x$ of length at most $k$ ?

The general version of this problem is $P S P A C E$-complete by a reduction from LINEAR BOUNDED AUTOMATON ACCEPTANCE (see Garey and Johnson [81, AL20]). This problem is $W[1]$-complete by a reduction from CLIQUE (Downey et al. [62], [63].)

\section{SHORT TURING MACHINE ACCEPTANCE}

Instance: A nondeterministic Turing machine $M$ operating on alphabet $\Sigma$, a word $x \in \Sigma^{*}$. Parameter: A positive integer $k$.

Question: Is there a computation of $M$ on input $x$ that reaches an accept state in at most $k$ steps?

The general version of this problem is undecidable (see for example Hopcroft and Ullman [88]). This problem is $W$ [1]-complete by a reduction from CLIQUE (Downey et al. [63], Cesati [44]). In FPT if either the size of the alphabet or the number of nondeterministic transition possibilities out of a given state is bounded.

\section{SHORT POST CORRESPONDENCE}

Instance: A Post system $\Pi$.

Parameter: A positive integer $k$.

Question: Is there a length $k$ solution for $\Pi$ ?

The classical POST CORRESPONDENCE problem is a well-known undecidable problem. This problem is $W[1]$-complete by a reduction from SHORT UNRESTRICTED GRAMMAR DERIVATION (Cai et al. [40].)

\section{SHORT UNRESTRICTED GRAMMAR DERIVATION}

Instance: An unrestricted phrase-structure grammar $G$, a word $x$.

Parameter: A positive integer $k$.

Question: Is there a $G$-derivation of $x$ of length at most $k$ ?

The general version of this problem is undecidable (see for example Hopcroft and Ullman [88]. This problem is $W[1]$-complete by a reduction from CLIQUE (Cai et al. [40]).

\section{SQUARE TILING}

Instance: A set $C$ of "colors," a collection $T \subseteq C^{4}$ of "tiles" (where $\langle a, b, c, d\rangle$ denotes a tile whose top, right, bottom, and left sides are colored $a, b, c$, and $d$, respectively), a positive integer $k \leq C$.

Parameter: $k$

Question: Is there a tiling of an $k \times k$ square using the tiles in $T$, i.e., an assignment of a tile $A(i, j) \in T$ to each ordered pair $i, j, 1 \leq i \leq k, 1 \leq j \leq k$, such that (1) if $f(i, j)=$ $\langle a, b, c, d\rangle$ and $f(i+1, j)=\left\langle a^{\prime}, b^{\prime}, c^{\prime}, d^{\prime}\right\rangle$, then $a=c^{\prime}$, and (2) if $f(i, j)=\langle a, b, c, d\rangle$ and $f(i, j+1)=\left\langle a^{\prime}, b^{\prime}, c^{\prime}, d^{\prime}\right\rangle$, then $b=d^{\prime}$. 
The general version of this problem is $N P$-complete by a reduction from DIRECTED HAMILTON PATH (see Garey and Johnson [81, GP13]). This problem is W[1]-complete by Cai et al. [40], Downey and Fellows [61].

\section{VAPNIK-CHERVONENKIS (VC) DIMENSION}

Instance: A family of subsets $F$ of a base set $X$.

Parameter: A positive integer $k$.

Question: Is the VC dimension of $F$ at least $k$ ? (The VC dimension of a family of subsets $F$ of a base set $X$ is the maximum cardinality of a set $S \subseteq X$ such that for each subset $S^{\prime} \subseteq S$, $\exists Y \in F$ such that $S \cap Y=S^{\prime}$.)

The general version of this problem is LOGSNP-complete (Papadimitriou and Yannakakis [100]). This problem is $W[1]$-complete by a reduction from CLIQUE (Downey, Evans, and Fellows [54]). Membership of $W[1]$ is proven by a generic reduction in [59].

\section{WEIGHTED $q$-CNF SATISFIABILITY}

Instance: A $q$-CNF formula $X$, i.e., a CNF formula such that each clause has no more than $q$ literals.

Parameter: A positive integer $k$.

Question: Does $X$ have a satisfying assignment of weight $k$ ?

This problem is $W[1]$-complete by a reduction from INDEPENDENT SET (Downey and Fellows [56]).

\subsection{W[1]-hard, in $W[2]$.}

\section{PERFECT CODE}

Instance: A graph $G=(V, E)$.

Parameter: A positive integer $k$.

Question: Does $G$ have a $k$-element perfect code? (A perfect code is a set of vertices $V^{\prime} \subseteq V$ with the property that for each vertex $v \in V$ there is precisely one vertex in $N[v] \cap V^{\prime}$.)

This problem is $W[1]$-hard by a reduction from INDEPENDENT SET and in $W[2]$ by (Downey and Fellows [56]). We believe that it may be difficult intermediate between $W[1]$ and $W[2]$.

\section{WEIGHTED EXACT CNF SATISFIABILITY}

Instance: A boolean expression $E$ in conjunctive normal form.

Parameter: A positive integer $k$.

Question: Is there a truth assignment of weight $k$ to the variables of $E$ that makes exactly one literal in each clause of $E$ true?

This problem is $W$ [1]-hard by a reduction from PERFECT CODE and in $W$ [2] by (Downey and Fellows [56]). Equivalent to PERFECT CODE (Downey and Fellows [56]). A related problem is UNIQUE WEIGHTED CNF SATISFIABILITY below.

\section{UNIQUE WEIGHTED CNF SATISFIABILITY}

Instance: A boolean CNF formula $X$.

Parameter: A positive integer $k$.

Question: Is there a unique weight $k$ satisfying assignment for $X$ ?

Clearly this is in $W[2]$. There are obvious versions of the above for normalized satisfiability at any level of the $W$-Hierarchy. For the CNF situation above, the problem is clearly in the natural analogue of $D_{p}$. The reader should recall that $D_{p}$ consists of the class of languages $L$ that can be expressed as the interchapter of a language in NP and one in co-NP. Clearly we 
can similarly define $D_{p}[2]$ (or, more generally $D_{p}[t]$ ) as for $D_{p}$ but with $W[2]$ in place of $N P$. Then this problem is in $D_{p}$ [2], as we see below. Before we prove this we remark that using the Valiant-Vazirani technique [114] we can show that UNIQUE WEIGHTED CNF SATISFIABILITY for arbitary boolean formulae is the same as WEIGHTED CNF SATISFIABILITY for boolean formulae under randomized reductions. However, on the face of it, it is not clear if this is true for any finite level of the $W$-Hierarchy since for instance weight is lost in the [VV] proof. Nevertheless using a new argument based on coding theory (Hadamard codes) together with Ken Regan the authors [64] have shown that a weighted version of [114] holds for all $t \geq 2$. This seems a very fruitful area to analyse.

UNIQUE WEIGHTED CNF SATISFIABILITY is in $D_{p}$ [2]

It suffices to describe how to say that a CNF expression has at least two satisfying expressions in $W[2]$. Let $C$ be the circuit corresponding to $X$. Take two copies of $C$. Add $O|X|$ many gates to express the fact that the first copy of $C$ has a satisfying assignment different from the second. Now for $k$ choose $q$ and $r$ appropriately and take $q$ copies of the the left circuit and $r$ copies of the right. Add new gates to express the fact that the inputs of the left $q$ must all be equal and the fact that the inputs of the right $r$ must all be equal. Now accept $C$ if the new circuit has a weight $(q+r) k$ accepting input. Then for the correct choice of $(q, r)$, depending only on $k, C$ has two or more accepting inputs if and only if the new circuit has one of weight $(q+r) k$.

We remark that this seems to be where UNIQUE DOMINATING SET would lie. We do not at present know if there are $D_{p}[t]$ complete problems.

5.6. $W[1]-h a r d$, in $W[P]$.

\section{PERMUTATION GROUP FACTORIZATION}

Instance: A set $A$ of permutations $A \subseteq S_{n}, x \in S_{n}$.

Parameter: A positive integer $k$.

Question: Does $x$ have a factorization of length $k$ over $A$ ?

This problem is $W[1]$-hard by a reduction from PERFECT CODE and in $W[P]$ by Cai et al. [40].

\section{SUBSET SUM}

Instance: A set of integers $X=\left\{x_{1}, \ldots, x_{n}\right\}$, integers $s, k$.

Parameter: $k$

Question: Is there a subset $X^{\prime} \subseteq X$ of cardinality $k$ such that the sum of the integers in $X^{\prime}$ equals $s$ ?

The general version of this problem is $N P$-complete by a reduction from Partition (see Garey and Johnson [81, SP13]). This problem is $W[1]$-hard by a reduction from PERFECT CODE Downey and Fellows [56] and in $W[P]$ by Fellows and Koblitz [73]. Not known to belong to $W[t]$ for any $t$.

\subsection{W[1]-hard.}

\section{$\alpha$-BALANCED SEPARATOR}

Instance: A graph $G=(V, E)$.

Parameter: A positive integer $k$.

Question: Does there exist a set of vertices $S,|S| \leq k$, such that every component of $G[V-S]$ has at most $\alpha|V|$ vertices?

This problem is $W[1]$-hard by a reduction from CLIQUE by Kaplan and Shamir [90]. This problem is $W[1]$-hard for every fixed $\alpha$. 


\section{COLORED PROPER INTERVAL GRAPH COMPLETION}

Instance: A graph $G=(V, E)$, a vertex coloring $c: V \rightarrow\{1, \ldots, k\}$.

Parameter: $k$

Question: Does there exist a proper interval supergraph of $G$ which respects $c$ ?

This problem is $W[1]$-hard by a reduction from INDEPENDENT SET by Kaplan and Shamir [90].

\section{COLORED UNIT INTERVAL GRAPH COMPLETION}

Instance: A graph $G=(V, E)$, a vertex coloring $c: V \rightarrow\{1, \ldots, k\}$.

Parameter: $k$

Question: Does there exist a graph $G^{\prime}=\left(V, E^{\prime}\right)$ such that $E^{\prime} \supseteq E, G^{\prime}$ is a unit interval graph and $G^{\prime}$ is properly colored by $c$ ?

The general version of this problem is NP-complete by Kaplan and Shamir [90]. This problem is $W[1]$-hard by Kaplan and Shamir [90].

\section{EXACT CHEAP TOUR}

Instance: A weighted graph $G=(V, E)$, a weight function $w: E \rightarrow \mathbf{Z}$.

Parameter: A positive integer $k$.

Question: Is there a tour through at least $k$ nodes of $G$ of cost exactly $S$ ?

The general version of this problem is $N P$-complete by a reduction from HAMILTON CIRCUIT (see Garey and Johnson [81, ND22]). This problem is $W$ [1]-hard by [56]. See the related SHORT CHEAP TOUR problem.

\section{INVMAX}

Instance: A circuit $\mathrm{C}$, an initial configuration $\operatorname{conf}_{0}$ describing the placing of inverters on connections between gates in $G$.

Parameter: A positive integer $k$.

Question: Is there a subset $A$ of the gates in $G$ to which DeMorgan's rules can be applied such that the resulting circuit will have at least $k$ gates without any inverters attached to their output lines?

The general version of this problem is $N P$-complete by a reduction from INDEPENDENT SET (Simon [109]). This problem is $W$ [1]-hard by the same reduction.

\section{PROPER INTERVAL SANDWICH WITH BOUNDED CLIQUE SIZE}

Instance: A sandwich instance $S=\left(V, E^{1}, E^{3}\right)$.

Parameter: A positive integer $k$.

Question: Does there exist a sandwich $G$ for $S$ which is a proper interval graph such that the size of largest clique is at most $k$ ?

The general version of this problem is $N P$-complete by Kaplan and Shamir [90]. This problem is $W[1]$-hard by a reduction from COLORED INTERVAL GRAPH COMPLETION by Kaplan and Shamir [90].

\section{REACHABILITY DISTANCE FOR VECTOR ADDITION SYSTEMS (PETRI NETS)}

Instance: A set $T$ of $m$ length $n$ integer-valued vectors $T=\left\{x^{i}=\left(x_{1}^{i}, \ldots, x_{n}^{i}\right): 1, \leq i \leq m\right\}$, a nonnegative starting vector $s=\left(s_{1}, \ldots, s_{n}\right)$, a nonnegative target vector $t=\left(t_{1}, \ldots, t_{n}\right)$.

Parameter: A positive integer $k$.

Question: Is there a choice of $k$ indices $i_{1}, \ldots, i_{k}, 1 \leq i_{j} \leq m$ for $j=1, \ldots, k$ such that $t=s+\sum_{j=1}^{k} x^{i_{j}}$ and such that every intermediate sum is nonnegative in each component, that is, $s_{r}+\sum_{j=1}^{q} x_{r}^{i_{j}} \geq 0$ for $q=1, \ldots, k$ and $r=1, \ldots, n$ ?

This problem is $W[1]$-hard by a reduction from CLIQUE (Downey et al. [63]). 


\section{SHORT $l$ TAPE NDTM COMPUTATION (I)}

Instance: An $l$-tape nondeterministic Turing machine $M$ operating on alphabet $\Sigma$, a word $x \in \Sigma^{*}$.

Parameter: A positive integer $k$.

Question: Is there a computation of $M$ on input $x$ that reaches an accept state in at most $k$ steps?

The general version of this problem is undecidable (see Hopcroft and Ullman [88]). This problem is $W[1]$-hard by a reduction from SHORT TURING MACHINE COMPUTATION (Cesati [44]).

\section{SHORT $l$ TAPE NDTM COMPUTATION (II)}

Instance: An $l$-tape nondeterministic Turing machine $M$ operating on alphabet $\Sigma$, a word $x \in \Sigma^{*}$, a positive integer $k$.

Parameter: $k, l$

Question: Is there a computation of $M$ on input $x$ that reaches an accept state in at most $k$ steps?

The general version of this problem is undecidable (see Hopcroft and Ullman [88]). This problem is $W[1]$-hard by a reduction from SHORT TURING MACHINE COMPUTATION (Cesati[44]).

\section{SUBSET PRODUCT}

Instance: A set of integers $X=\left\{x_{1}, \ldots, x_{n}\right\}$, integers $a, m, k$.

Parameter: $k$

Question: Is there a subset $X^{\prime} \subseteq X$ of cardinality $k$ such that the product of the integers in $X^{\prime}$ is congruent to $a \bmod m$ ?

The general version of this problem is $N P$-complete by a reduction from X3C (see Garey and Johnson [81, SP14]). This problem is $W[1]$-hard by a reduction from PERFECT CODE by Fellows and Koblitz [72], [73].

\section{COLORED GRAPH AUTOMORPHISM}

Instance: A 2-colored (bipartite) graph $G$.

Parameter: A positive integer $k$.

Question: Is there an automorphism preserving colors moving exactly $k$ blue vertices?

$W[1]$-hard by a reduction from ANTIMONOTONE 2SAT (Downey and Fellows [61]).

\section{8. $W[2]-c o m p l e t e$.}

\section{DOMINATING SET}

Instance: A graph $G=(V, E)$.

Parameter: A positive integer $k$.

Question: Is there a set of $k$ vertices $V^{\prime} \subseteq V$ with the property that every vertex of $G$ either belongs to $V^{\prime}$ or has a neighbor in $V^{\prime}$ ?

The general version of this problem is $N P$-complete by a reduction from VERTEX COVER (see Garey and Johnson [81, GT2]). This problem is $W[2]$-complete by a reduction from WEIGHTED CNF SATISFIABILITY (Downey and Fellows [55], this paper). Fixedparameter tractable for planar graphs (Downey and Fellows [59]). Problem is $W$ [2]-hard if the dominating set $V^{\prime}$ is required to be either connected or total, i.e., for each vertex in $V$ there is an edge to some vertex in $V^{\prime}$ (Bodlaender and Kratsch [30]. 


\section{HITTING SET}

Instance: A finite family of sets $S=S_{1}, \ldots, S_{n}$ comprised of elements from $U=\left\{u_{1}, \ldots, u_{m}\right\}$. Parameter: A positive integer $k$.

Question: Is there a subset $T \subseteq U$ of size $k$ such that for all $S_{i} \in S, S_{i} \cap T \neq \emptyset$ ?

The general version of this problem is $N P$-complete by a reduction from VERTEX COVER (see Garey and Johnson [81, SP8]). This problem is $W[2]$-complete by a reduction from SET COVER (Ausiello, D'Atri, and Protasi [11], Wareham [117]).

\section{INDEPENDENT DOMINATING SET}

Instance: A graph $G=(V, E)$.

Parameter: A positive integer $k$.

Question: Is there a set of $k$ vertices $V^{\prime} \subseteq V$ that is both an independent set and a dominating set in $G$ ?

The general version of this problem is $N P$-complete by a reduction from VERTEX COVER (see Garey and Johnson [81, GT2]). This problem is $W[2]$-complete by Downey and Fellows [55]. Fixed-parameter tractable for planar graphs (Downey and Fellows [59]).

\section{SET COVER}

Instance: A finite family of sets $S=S_{1}, \ldots, S_{n}$.

Parameter: A positive integer $k$.

Question: Is there a subset $R \subseteq S$ whose union is all elements in the union of $S$ ?

The general version of this problem is $N P$-complete by a reduction from $\mathrm{X} 3 \mathrm{C}$ (see Garey and Johnson [81, SP5]). This problem is $W[2]$-complete by a reduction from DOMINATING SET by Paz and Moran [101], Wareham [117].

\section{TOURNAMENT DOMINATING SET}

Instance: A tournament $T$.

Parameter: A positive integer $k$.

Question: Does $T$ have a dominating set of cardinality at most $k$ ?

The general version of this problem is LOGSNP-complete (Papadimitriou and Yannakakis [100]). This problem is $W[2]$-complete by a reduction from DOMINATING SET (Downey and Fellows [59]).

\section{WEIGHTED BINARY INTEGER PROGRAMMING}

Instance: A binary matrix $A$, a binary vector $\mathbf{b}$.

Parameter: A positive integer $k$.

Question: Does $A \cdot \mathbf{x} \geq \mathbf{b}$ have a binary solution of weight $k$ ?

The general version of this problem is $N P$-complete by a reduction from 3SAT (see Garey and Johnson [81, MP1]). This problem is $W[2]$-complete by a reduction from MONOTONE WEIGHTED CNF SATISFIABILITY (Downey and Fellows, this paper). The problem WEIGHTED EXACT BINARY INTEGER PROGRAMMING asks that equality hold is hard for $W[1]$.

\section{9. $W[2]$-hard, in $W[P]$.}

\section{MONOCHROME CYCLE COVER}

Instance: An edge-colored graph $G=(V, E)$.

Parameter: A positive integer $k$.

Question: Is there a set of $k$ vertices $V^{\prime} \subseteq V$ with the property that every monochrome cycle in $G$ contains a vertex in $V^{\prime}$ ? 
This problem is $W[2]$-hard and in $W[P]$ by Downey and Fellows [61]. Not known to belong to $W[t]$ for any $t$, but easily shown to be in $W[P]$.

\section{MONOID FACTORIZATION}

Instance: A set $A$ of self-maps on [ $n$ ], a self-map $h$.

Parameter: A positive integer $k$.

Question: Is there a factorization of $h$ of length $k$ over $A$ ?

This problem is $W[2]$-hard by a reduction from DOMINATING SET and in $W[P]$ by Cai et al. [40].

\subsection{W[2]-hard.}

\section{DOMINATING CLIQUE}

Instance: A graph $G=(V, E)$.

Parameter: A positive integer $k$.

Question: Is there a set of $k$ vertices $V^{\prime} \subseteq V$ that forms a complete subgraph of $G$ and is also a dominating set for $G$ ?

This problem is $W[2]$-hard by a reduction from DOMINATING SET (Bodlaender and Kratsch [30]). Problem is in FPT if $V^{\prime}$ is also required to be efficient, i.e., each vertex not in $V^{\prime}$ is dominated by exactly one vertex in $V^{\prime}$ (Bodlaender and Kratsch [30]).

\section{LONGEST COMMON SUBSEQUENCE II}

Instance: A set of $k$ strings $X_{1}, \ldots, X_{k}$ over an alphabet $\Sigma$, a positive integer $m$.

Parameter: $k, m$

Question: Is there a string $X \in \Sigma^{*}$ of length at least $m$ that is a subsequence of $X_{i}$ for $i=1, \ldots, k$ ?

The general version of this problem is NP-complete by a reduction from VERTEX COVER (see Garey and Johnson [81, SR 10]). This problem is $W[2]$-hard by a reduction from DOMINATING SET (Bodlaender, et al. [25]).

\section{MAXIMAL IRREDUNDANT SET}

Instance: A graph $G=(V, E)$.

Parameter: A positive integer $k$.

Question: Is there a set $V^{\prime} \subseteq V$ of cardinality $k$ such that (1) each vertex $u \in V^{\prime}$ has a private neighbor and (2) $V^{\prime}$ is not a proper subset of any $V^{\prime \prime} \subseteq V$ which also has this property? (A private neighbor of a vertex $u \in V^{\prime}$ is a vertex $u^{\prime}$ (possibly $u^{\prime}=u$ ) with the property that for every vertex $v \in V^{\prime}, u \neq v, u^{\prime} \notin N[v]$.)

This problem is $W[2]$-hard by a reduction from DOMINATING SET (Bodlaender and Kratsch [30]). Originally proven to be $W[1]$ hard by Downey and Fellows (see [61]).

\section{PRECEDENCE CONSTRAINED $k$-PROCESSOR SCHEDULING}

Instance: A set $T$ of unit-length tasks, a partial order $\prec$ on $T$, a positive integer deadline $D$, a number of processors $k$.

Parameter: $k$

Question: Is there a map $f: T \rightarrow\{1, \ldots, D\}$, such that for all $t, t^{\prime} \in T, t \prec t^{\prime}$ implies $f(t)<f\left(t^{\prime}\right)$, and for all $i, 1 \leq i \leq D,\left|f^{-1}(i)\right| \leq k$ ?

The general version of this problem is Open (Garey and Johnson [81, OPEN8]). This problem is $W[2]$-hard by a reduction from DOMINATING SET (Bodlaender, Fellows, and Hallett [28]). 


\section{STEINER TREE}

Instance: A graph $G=(V, E)$, a set $S$ of at most $k$ vertices in $V$, an integer $m$.

Parameter: $k, m$

Question: Is there a set of vertices $T \subseteq V-S$ such that $|T| \leq m$ and $G[S \cup T]$ is connected?

The general version of this problem is $N P$-complete by a reduction from EXACT COVER (see Garey and Johnson [81, ND12, GKR]). This problem is $W[2]$-hard by a reduction from DOMINATING SET (Bodlaender and Kratsch [30]).

\subsection{1. $W[3]-h a r d$, in $W[4]$.}

\section{DOMINATING THRESHOLD SET}

Instance: A graph $G=(V, E)$.

Parameter: Positive integers $k, r$.

Question: Is there a set $V^{\prime} \subseteq V$ of at most $k$ vertices such that for every vertex $u, N[u]$ contains at least $r$ elements of $V^{\prime}$ ?

This problem is $W[3]$-hard and in $W[4]$ by Fellows [69].

\subsection{2. $W[t]$-complete.}

\section{WEIGHTED $t$-NORMALIZED SATISFIABILITY}

Instance: A $t$-normalized boolean expression $X$.

Parameter: A positive integer $k$.

Question: Does $X$ have a satisfying truth assignment of weight $k$ ?

This problem is $W[t]$-complete by Downey and Fellows (this paper).

\section{$\leq k$ WEIGHTED $t$-NORMALIZED SATISFIABILITY}

Instance: A boolean formula $X$.

Parameter: A positive integer $k$.

Question: Does $X$ have a satisfying assignment of weight $\leq k$ ?

For $t \geq 2$ this problem is $W[t]$-complete by Cai and Chen [37]-[39]. This fact also follows by the main lemma of this paper. For $t=1$ the problem is in FPT.

\subsection{3. $W[t]$-hard, for all $t$, in $W[P]$.}

\section{SHORT PHONOLOGICAL SEGMENTAL DECODING}

Instance: An integer $\mathrm{k}$, a simplified segmental grammar $s=\left(F, S, D, R, c_{p}, C\right)$ such that the number of mutually exclusive rule sets in $R,\left|R_{m . e .}\right|$, is at most $k$, string $s \in S^{+}$.

Parameter: $k$

Question: Is there a string $u \in D$ such that $g(u)=s$ ?

The general version of this problem is $N P$-complete by a reduction from CLIQUE Ristad [105]. This problem is $W[t]$-hard by a reduction from WEIGHTED $t$-NORMALIZED SATISFIABILITY and in $W[P]$ by Downey et al. [63]. See Downey et al. [63] and Ristad [105] for definitions of simplified segmental grammars. The same proof also implies the $W[t]$-hardness and membership in $W[P]$ of SHORT PHONOLOGICAL SEGMENTAL ENCODING.

\subsection{4. $W[t]$-hard, for all $t$.}

\section{BANDWIDTH}

Instance: A graph $G=(V, E)$.

Parameter: A positive integer $k$. 
Question: Is there a 1:1 linear layout $f: V \rightarrow\{1, \ldots,|V|\}$ such that $u v \in E$ implies $|f(u)-f(v)| \leq k$ ?

The general version of this problem is NP-complete by a reduction from 3PARTITION (see Garey and Johnson [81, ND40]). This problem is $W[t]$-hard by a reduction from UNIFORM EMULATION ON A PATH (Bodlaender, Fellows, and Hallett [28]). Remains $W[t]$-hard for all $t$ when given graph is directed and layout must respect arc direction, or when given graph is a tree ([28]). The related problem CUTWIDTH is FPT (Fellows and Langston [74]).

\section{COLORED CUTWIDTH}

Instance: A graph $G=(V, E)$, an edge coloring $c: E \rightarrow\{1, \ldots, r\}$.

Parameter: A positive integer $k$.

Question: Is there a $1: 1$ linear layout $f: V \rightarrow\{1, \ldots,|V|\}$ such that for each color $j \in$ $\{1, \ldots, k\}$ and for each $i, 1 \leq i \leq|V|-1$, we have $\mid\{u v: c(u v)=j$ and $f(u) \leq i$ and $f(v) \geq i+1\} \mid \leq r ?$

This problem is $W[t]$-hard by a reduction from LONGEST COMMON SUBSEQUENCE II (Bodlaender, Fellows, and Hallett [28]).

DOMINO TREEWIDTH

Instance: A graph $G=(V, E)$.

Parameter: A positive integer $k$.

Question: Is the domino treewidth of $G$ at most $k$ ?

The general version of this problem is $N P$-complete by a reduction from LONGEST COMMON SUBSEQUENCE II by Bodlaender and Engelfriet [27]. This problem is $W[t]-$ hard by the same reduction.

\section{FEASIBLE REGISTER ASSIGNMENT}

Instance: A directed acyclic graph $G=(V, E)$, a positive integer $k$, a register assignment $r: V \rightarrow\left\{R_{1}, \ldots, R_{k}\right\}$.

Parameter: $k$

Question: Is there a linear ordering $f$ of $G$, and a sequence $S_{0}, S_{1}, \ldots, S_{|V|}$ of subsets of $V$, such that $S_{0}=\emptyset, S_{|V|}$ contains all vertices of in-degree 0 in $G$, and for all $i, 1 \leq i \leq|V|$, $f^{-1}(i) \in S_{i}, S_{i}-\left\{f^{-1}(i)\right\} \subseteq S_{i-1}$ and $S_{i-1}$ contains all vertices $u$ for which $\left(f^{-1}(i), u\right) \in E$, and for all $j, 1 \leq j \leq k$, there is at most one vertex $u \in S_{i}$ with $r(u)=R_{j}$ ?

The general version of this problem is NP-complete by a reduction from 3SAT (see Garey and Johnson [81, PO2]). This problem is $W[t]$-hard by a reduction from LONGEST COMMON SUBSEQUENCE II (Bodlaender, Fellows, and Hallett [28].)

INTERVALIZING COLORED GRAPHS (DNA PHYSICAL MAPPING)

Instance: A graph $G=(V, E)$, vertex coloring $c: V \rightarrow\{1, \ldots, k\}$.

Parameter: $k$

Question: Does there exist a supergraph $G^{\prime}=\left(V, E^{\prime}\right)$ where $E \subseteq E^{\prime}$ and $G^{\prime}$ is properly colored by $c$ and is an interval graph?

The general version of this problem is NP-complete by a reduction from BETWEENNESS (Golumbic, Kaplan, and Shamir [85]) and INDEPENDENT SET (Fellows, Hallett, and Wareham [71]). This problem is $W[t]$-hard by a reduction from COLORED CUTWIDTH (Bodlaender, Fellows, and Hallett [28]). No polynomial time algorithm is known for fixed $k$.

LONGEST COMMON SUBSEQUENCE II

Instance: A set of $k$ strings $X_{1}, \ldots, X_{k}$ over an alphabet $\Sigma$, a positive integer $m$. 
Parameter: $k$

Question: Is there a string $X \in \Sigma^{*}$ of length at least $m$ that is a subsequence of $X_{i}$ for $i=1, \ldots, k$ ?

The general version of this problem is $N P$-complete by a reduction from VERTEX COVER (see Garey and Johnson [81, SR 10]). This problem is $W[t]$-hard by a reduction from MONOTONE WEIGHTED $t$-NORMALIZED SATISFIABILITY (Bodlaender, Fellows, and Hallett [28]).

\section{LONGEST COMMON SUBSEQUENCE III}

Instance: A set of $k$ strings $X_{1}, \ldots, X_{k}$ over an alphabet $\Sigma$, a positive integer $m$.

Parameter: $k,|\Sigma|$

Question: Is there a string $X \in \Sigma^{*}$ of length at least $m$ that is a subsequence of $X_{i}$ for $i=1, \ldots, k$ ?

The general version of this problem is $N P$-complete by a reduction from VERTEX COVER (see Garey and Johnson [81, SR 10]). This problem is $W[t]$-hard by a reduction from Bodlaender et al. [24].

\section{PROPER INTERVAL GRAPH COMPLETION PROBLEM WITH MINIMUM CLIQUE SIZE}

Instance: A graph $G=(V, E)$.

Parameter: A positive integer $k$.

Question: Does there exist a graph $G^{\prime}=\left(V, E^{\prime}\right), E^{\prime} \supseteq E$, such that $G^{\prime}$ is an interval graph and has minimum clique size $k$ ?

The general version of this problem is NP-complete by Kaplan and Shamir [90]. This problem is $W[t]$-hard by Kaplan and Shamir [90]. Equivalent to the BANDWIDTH problem by Kaplan and Shamir [90] (also Hallett [87]).

\section{PROPER INTERVAL GRAPH COMPLETION WITH BOUNDED CLIQUE SIZE} Input: A graph $G$.

Parameter: A positive integer $k$.

Question: Is there a $G^{\prime} \supseteq G$ which is a proper interval graph and has clique size at most $k$ ?

The general problem is $N P$-complete and the parameterized problem hard for $W[t]$ for all $t$ by Kaplan, Shamir, and Tarjan [91].

\section{RESTRICTED COMPLETION TO A PROPER INTERVAL GRAPH WITH BOUNDED CLIQUE SIZE \\ Input: A graph $G$ together with a set $E$ of edges prohibited from $G^{\prime}$ below.}

Parameter: A positive integer $k$.

Question: Is there a $G^{\prime} \supset G$ which is a proper interval graph and has clique size at most $k$, and $G^{\prime}$ has no edges from $E$ ?

\section{TRIANGULATING COLORED GRAPHS}

Instance: A graph $G=(V, E)$, vertex coloring $c: V \rightarrow\{1, \ldots, k\}$.

Parameter: $k$

Question: Does there exist a supergraph $G^{\prime}=\left(V, E^{\prime}\right)$ where $E \subseteq E^{\prime}$ and $G^{\prime}$ is properly colored by $c$ and $G^{\prime}$ is triangulated?

The general version of this problem is $N P$-complete by a reduction from INDEPENDENT SET (Bodlaender, Fellows, and Hallett [28]). This problem is $W[t]$-hard by a reduction from LONGEST COMMON SUBSEQUENCE II [28]. 


\section{UNIFORM EMULATION ON A PATH}

Instance: A graph $G=(V, E)$.

Parameter: A positive integer $k$.

Question: Does there exist a function $f: V \rightarrow\{1, \ldots,|V| / k\}$ such that for all $u v \in E$ implies $|f(u)-f(v)| \leq 1$ and for all $i,\left|f^{-1}(i)\right| \leq k$ ?

This problem is $W[t]$-hard by a reduction from MONOTONE WEIGHTED $t$-NORMALIZED SATISFIABILITY (Bodlaender, Fellows, and Hallett [28]). Remains W[ $t$ ]-hard for all $t$ when given graph is a tree [28].

\subsection{5. $W[P]$-complete.}

$k$-BASED TILING

Instance: A tiling system with distinguished tiles.

Parameter: A positive integer $k$.

Question: Is there a tiling of the $n \times n$ plane using the tiling system and starting with exactly $k$ distinguished tiles in a line ?

Reduction consists of a generic simulation of a Turing machine (see Downey and Fellows [61]).

\section{$k$-INDUCED 3CNF SATISFIABILITY}

Instance: A $3 \mathrm{CNF}$ formula $\varphi$.

Parameter: A positive integer $k$.

Question: Is there a set of $k$ variables and a truth table assignment to those variables that causes $\varphi$ to unravel?

This problem is $W[P]$-complete by a reduction from CHAIN REACTION CLOSURE (Abrahamson, Downey, and Fellows [2]) (also Abrahamson et al. [3]).

\section{$k$-INDUCED SATISFIABILITY}

Instance: A boolean formula $\varphi$.

Parameter: A positive integer $k$.

Question: Is there a set of $k$ variables and a truth table assignment to those variables that causes $\varphi$ to unravel?

This problem is $W[P]$-complete by a reduction from $k$-INDUCED 3CNF SATISFIABILITY (Abrahamson, Downey, and Fellows [2]).

\section{CHAIN REACTION CLOSURE}

Instance: A directed graph $D=(V, A)$.

Parameter: A positive integer $k$.

Question: Does there exist a set $V^{\prime}$ of $k$ vertices of $D$ whose chain reaction closure is $D$ ? (A chain reaction closure of $V^{\prime}$ is the smallest superset $S$ of $V^{\prime}$ such that if $u, u^{\prime} \in S$ and $\operatorname{arcs} u x$, $u^{\prime} x$ are in $D$ then $x \in S$.)

This problem is $W[P]$-complete by a reduction from WEIGHTED MONOTONE CIRCUIT SATISFIABILITY (Abrahamson, Downey, and Fellows [2]).

\section{DEGREE 3 SUBGRAPH ANNIHILATOR}

Instance: A graph $G=(V, E)$.

Parameter: A positive integer $k$.

Question: Is there a set of $k$ vertices $V^{\prime} \subseteq V$ such that $G-V^{\prime}$ has no subgraph of minimum degree 3 ? 
This problem is $W[P]$-complete by a reduction from WEIGHTED MONOTONE CIRCUIT SATISFIABILITY (Abrahamson, Downey, and Fellows [2]).

\section{LINEAR INEQUALITIES}

Instance: A system of linear inequalities.

Parameter: A positive integer $k$.

Question: Can we delete $k$ of the equalities and get a system that is consistent over the rationals?

This problem is $W[P]$-complete by a reduction from WEIGHTED MONOTONE CIRCUIT SATISFIABILITY taken from Abrahamson et al. [3] (Abrahamson, Downey, and Fellows [2]).

\section{MINIMUM AXIOM SET}

Instance: A finite set $S$ of sentences, an implication relation $R$ consisiting of pairs $(A, t)$ where $A \subseteq S$ and $t \in S$.

Parameter: A positive integer $k$.

Question: Is there a set $S_{0} \subseteq S$ with $\left|S_{0}\right| \leq k$ and a positive integer $n$ such that if we define $S_{i}, 1 \leq i \leq n$, to consist of exactly those $t \in S$ for which either $t \in S_{i-1}$ or there exists a set $U \subseteq S_{i-1}$ such that if $(U, t) \in R$ then $S_{n}=S$ ?

The general version of this problem is $N P$-complete by a reduction from $\mathrm{X} 3 \mathrm{C}$ (see Garey and Johnson [81, L017]). This problem is $W[P]$-complete by a reduction from WEIGHTED CIRCUIT SATISFIABILITY (Downey et al. [63] and Abrahamson, Downey, and Fellows [2]).

\section{SHORT CIRCUIT SATISFIABILITY}

Instance: A boolean circuit $C$ with $n$ gates and at most $k \log n$ inputs and one output.

Parameter: $k$

Question: Is there a setting of the inputs that cause $C$ to output 1 ?

This problem is $W[P]$-complete by a reduction from WEIGHTED CIRCUIT SATISFIABILITY (Abrahamson et al. [3], also Abrahamson, Downey, and Fellows [2]).

\section{SHORT SATISFIABILITY}

Instance: A formula $\varphi$ on $n$ variables, a list of at $\operatorname{most} k \log n$ variables of $\varphi$.

Parameter: $k$

Question: Is there any setting of the distinguished varaibles that causes $\varphi$ to unravel?

This problem is $W[P]$-complete by a reduction from SHORT CIRCUIT SATISFIABILITY (Abrahamson, Downey, and Fellows [2]).

\section{THRESHOLD STARTING SET}

Instance: A directed graph $D=(V, A)$.

Parameter: A positive integer $k$.

Question: Does $G$ have a starting set of size $k$ ? (A starting set is a set of vertices $V^{\prime} \subseteq V$ with the property that if we begin with a pebble on each of the vertices in $V^{\prime}$ and subsequently place pebbles on any vertex having at least $t$ incoming arcs from pebbled vertices then eventually every vertex of the graph is pebbled.)

This problem is $W[P]$-complete by a reduction from WEIGHTED MONOTONE CIRCUIT SATISFIABILITY (Abrahamson, Downey, and Fellows [2]).

WEIGHTED MONOTONE CIRCUIT SATISFIABILITY

Instance: A boolean monotone circuit $C$. 
Parameter: A positive integer $k$.

Question: Is there a weight $k$ input vector accepted by $C$ ?

This problem is $W[P]$-complete by a reduction from MINIMUM AXIOM SET (Downey et al. [63] and Abrahamson, Downey, and Fellows [2]).

\section{WEIGHTED PLANAR CIRCUIT SATISAFIABILITY}

Instance: A planar decision circuit $C$.

Parameter: A positive integer $k$.

Question: Does $C$ have a satisfying assignment of weight $k$ ?

This problem is $W[P]$-complete by a reduction from WEIGHTED CIRCUIT SATISFIABILITY (Abrahamson, Downey, and Fellows [2]).

Comment. The following classes are not discussed in this paper but are listed for completeness. They are analogues of the QBFSAT and PSPACE in some sense and correspond as we see to the complexity of $k$-move games. They are discussed at length in [1], [2]. In each case the first problem defines the class.

\subsection{6. $A W[S A T]$-complete.}

\section{PARAMETERIZED QBFSAT}

Instance: An integer $r$, a sequence $s_{1}, \ldots, s_{r}$ of pairwise disjoint sets of boolean variables, a boolean formula $X$ involving the variables $s_{1} \cup \cdots \cup s_{r}$, integers $k_{1}, \ldots, k_{r}$.

Parameter: $k_{1}, \ldots, k_{r}$

Question: Is it the case that there exists a size $k_{1}$ subset $t_{1}$ of $s_{1}$ such that for every size $k_{2}$ subset $t_{2}$ of $s_{2}$ there exists a size $k_{3}$ subset $t_{3}$ of $s_{3}$ such that ... (alternating quantifiers) such that, when the variables in $t_{1} \cup \cdots \cup t_{r}$ are made true and all other variables are made false, formula $X$ is true?

\section{PARAMETERIZED MONOTONE QBFSAT}

This is the same as PARAMETERIZED QBFSAT except that the formulae are monotone (Abrahamson, Downey, and Fellows [2]).

\section{PARAMETERIZED ANTIMONOTONE QBFSAT}

This is the same as PARAMETERIZED QBFSAT except that the formulae are antimonotome (Abrahamson, Downey, and Fellows [2]).

\subsection{7. $A W[S A T]$-hard.}

\section{COMPACT DTM COMPUTATION I}

Instance: A deterministic Turing machine $M$ operating on tape alphabet $\Sigma$, a word $x \in \Sigma^{*}$. Parameter: A positive integer $k$.

Question: Does $M$ on input $x$ accept after visiting at most $k$ work tape squares?

This problem is $A W[S A T]$-hard by a reduction from PARAMETERIZED QBFSAT $(r$, $k_{1}, \ldots, k_{r}$ ) (Cesati [44]).

\section{COMPACT DTM COMPUTATION II}

Instance: A deterministic Turing machine $M$ operating on tape alphabet $\Sigma$, a word $x \in \Sigma^{*}$. Parameter: A positive integer $k,|x|$.

Question: Does $M$ on input $x$ accept after visiting at most $k$ work tape squares?

This problem is $A W[S A T]$-hard by a reduction from PARAMETERIZED QBFSAT ( $r$, $\left.k_{1}, \ldots, k_{r}\right)($ Cesati [44]). 


\subsection{8. $A W[P]$-complete.}

\section{PARAMETERIZED QCSAT}

Instance: An integer $r$, a sequence $s_{1}, \ldots, s_{r}$ of pairwise disjoint sets of boolean variables, a circuit $X$ with the variables $s_{1} \cup \ldots \cup s_{r}$ as inputs, integers $k_{1}, \ldots, k_{r}$.

Parameter: $r, k_{1}, \ldots, k_{r}$.

Question: Is it the case that there exists a size $k_{1}$ subset $t_{1}$ of $s_{1}$ such that for every size $k_{2}$ subset $t_{2}$ of $s_{2}$ there exists a size $k_{3}$ subset $t_{3}$ of $s_{3}$ such that ... (alternating quantifiers) such that, when the inputs in $t_{1} \cup \cdots \cup t_{r}$ are set to 1 and all other inputs are set to 0 , circuit $X$ outputs 1 ?

\section{PARAMETERIZED MONOTONE QCSAT}

Instance: An integer $r$, a sequence $s_{1}, \ldots, s_{r}$ of pairwise disjoint sets of boolean variables, a montone circuit $X$ with the variables $s_{1} \cup \ldots \cup s_{r}$ as inputs, integers $k_{1}, \ldots, k_{r}$.

Parameter: $r, k_{1}, \ldots, k_{r}$.

Question: Is it the case that there exists a size $k_{1}$ subset $t_{1}$ of $s_{1}$ such that for every size $k_{2}$ subset $t_{2}$ of $s_{2}$ there exists a size $k_{3}$ subset $t_{3}$ of $s_{3}$ such that ... (alternating quantifiers) such that, when the inputs in $t_{1} \cup \cdots \cup t_{r}$ are set to 1 and all other inputs are set to 0 , circuit $X$ outputs 1 ?

This problem is $A W[P]$-complete by a reduction from PARAMETERIZED QCSAT (Abrahamson, Downey, Fellows, [2]) and MINIMUM AXIOM SET (Downey et al. [63]).

\section{PARAMETERIZED ANTIMONOTONE QCSAT}

This is the same as PARAMETERIZED QCSAT except the circuit must be antimonotone. $A W[P]$ complete by a reduction from PARAMETERIZED MONOTONE QCSAT. (Abrahamson, Downey, and Fellows [2]).

\subsection{9. $A W[P]$-hard.}

\section{COMPACT TURING MACHINE COMPUTATION}

Instance: A nondeterministic Turing machine $M$ operating on tape alphabet $\Sigma$, a word $x \in \Sigma^{*}$. Parameter: A positive integer $k$.

Question: Is there an accepting computation of $M$ on input $x$ that visits at most $k$ work tape squares?

This problem is $A W[P]$-hard by a reduction from PARAMETERIZED QCSAT $\left(r, k_{1}, \ldots\right.$, $k_{r}$ ) (Abrahamson, Downey, and Fellows [2]).

\section{COMPACT TURING MACHINE COMPUTATION II}

Instance: A nondeterministic Turing machine $M$ operating on tape alphabet $\Sigma$, a word $x \in \Sigma^{*}$. Parameter: A positive integer $k,|x|$.

Question: Is there an accepting computation of $M$ on input $x$ that visits at most $k$ work tape squares?

This problem is $A W[P]$-hard by a reduction from PARAMETERIZEDQCSAT $\left(r, k_{1}, \ldots\right.$, $k_{r}$ ) (Abrahamson, Downey, and Fellows [2]).

5.20. $A W\left[{ }^{*}\right]=A W[1]=A W[t]$-complete. Note that Abrahamson, Downey, and Fellows proved that $A W[*]=A W[2]$ in [1], [2]. This was recently improved to show that $A W[*]=$ $A W[1]$ (and in fact $N[*]=N[1]$ ) by Downey, Fellows, and Regan [64]. 


\section{PARAMETERIZED QBFSAT ${ }_{t}$}

Instance: An integer $r$, a sequence $s_{1}, \ldots, s_{r}$ of pairwise disjoint sets of boolean variables, a boolean formula $X$ involving the variables $s_{1} \cup \cdots \cup s_{r}$ which consists of $t$ alternating layers of conjunctions and disjunctions with negations applied only to variables, integers $k_{1}, \ldots, k_{r}$. Parameter: $r, k_{1}, \ldots, k_{r}$.

Question: Is it the case that there exists a size $k_{1}$ subset $t_{1}$ of $s_{1}$ such that for every size $k_{2}$ subset $t_{2}$ of $s_{2}$ there exists a size $k_{3}$ subset $t_{3}$ of $s_{3}$ such that ... (alternating quantifiers) such that, when the variables in $t_{1} \cup \cdots \cup t_{r}$ are made true and all other variables are made false, formula $X$ is true?

\section{UNITARY PARAMETERIZED QBFSAT $t_{t}$}

Instance: An integer $r$, a sequence $s_{1}, \ldots, s_{r}$ of pairwise disjoint sets of boolean variables, a boolean formula $X$ involving the variables $s_{1} \cup \cdots \cup s_{r}$ which consists of $t$ alternating layers of conjunctions and disjunctions with negations applied only to variables.

Parameter: A positive integer $k$.

Question: Is it the case that there exists a variable $t_{1}$ of $s_{1}$ such that for every variable $t_{2}$ of $s_{2}$ there exists a variable $t_{3}$ of $s_{3}$ such that ... (alternating quantifiers) such that, when the variables in $t_{1}, \ldots, t_{r}$ are made true and all other variables are made false, formula $X$ is true?

This problem is $A W[t]$-complete by Abrahamson, Downey, and Fellows [1], [2].

\section{SHORT GENERALIZED GEOGRAPHY}

Instance: A directed graph $D=(V, A)$, a specified vertex $v_{0} \in V$.

Parameter: A positive integer $k$.

Question: Does player one have a winning strategy in $k$ moves for the following game? Players alternately choose a new arc from $A$. The first arc chosen must have its tail at $v_{0}$ and each subsequently chosen arc must have its tail at the vertex that was the head of the previous arc. The first player unable to choose a new arc loses.

The general version of this problem is $P S P A C E$-complete by a reduction from QBFSAT (see Garey and Johnson [81, GP2]). This problem is $A W[*]$-complete by a reduction from UNITARY PARAMETERIZED QBFSAT ${ }_{t}$ (Abrahamson, Downey, and Fellows [2]).

\section{SHORT NODE KAYLES}

Instance: A graph $G=(V, E)$.

Parameter: A positive integer $k$.

Question: Does player one have a winning $k$-move strategy in the following game? That is, players pebble a vertex not adjacent to any pebbled vertex. The first player with no play loses. Player one plays first.

The general version of this problem is $P S P A C E$-complete by a reduction from QBFSAT (see Garey and Johnson [81, GP3]). This problem is $A W[*]$-complete by a reduction from UNITARY PARAMETERIZED QBFSAT ${ }_{t}$ (Abrahamson, Downey, and Fellows [2]).

\subsection{In $W[1]$.}

\section{IRREDUNDANT SET}

Instance: A graph $G=(V, E)$.

Parameter: A positive integer $k$.

Question: Is there a set $V^{\prime} \subseteq V$ of cardinality $k$ having the property that each vertex $u \in V^{\prime}$ has a private neighbor? (A private neighbor of a vertex $u \in V^{\prime}$ is a vertex $u^{\prime}$ (possibly $u^{\prime}=u$ ) with the property that for every vertex $v \in V^{\prime}, u \neq v, u^{\prime} \notin N[v]$.)

IRREDUNDANT SET is in $W[1]$ by Downey and Fellows [55]. 


\subsection{Open problems.}

\section{BOUNDED HAMMING WEIGHT DISCRETE LOGARITHM}

Instance: An $n$-bit prime, a generator $g$ of $F_{p}^{*}$, an element $a \in F_{p}^{*}$.

Parameter: A positive integer $k$.

Question: Is there a positive integer $x$ whose binary representation has at most $k 1$ 's (that is, $x$ has a Hamming weight of $k$ ) such that $a=g$ ?

Candidate for membership in randomized FPT (Fellows and Koblitz [72], [73]). This problem is of practical significance because the use of exponents of fairly small Hamming weight has been suggested in order to speed up cryptosystems based on discrete log (see [72], [73], and references).

\section{DIRECTED FEEDBACK VERTEX SET}

Instance: A directed graph $D=(V, A)$.

Parameter: A positive integer $k$.

Question: Is there a set $S$ of $k$ vertices such that each directed cycle of $G$ contains a member of $S$ ?

The general version of this problem is $N P$-complete by a reduction from VERTEX COVER (see Garey and Johnson [81, GT8]). This can be solved in $O\left(n^{k+1}\right)$ by brute force for each fixed $k$. A related problem DIRECTED FEEDBACK ARC SET asks for a set $A$ of at most $k$ arcs such that every directed cycle contains at least one arc from $A$. These problems can be shown to have the same $\leq_{m}^{s}$-degree. The undirected version of this problem is in FPT.

\section{IMMERSION ORDER TEST}

Instance: A graph $G=(V, E)$.

Parameter: A graph $H=\left(V^{\prime}, E^{\prime}\right)$.

Question: Is $H \leq_{i} G$ where $\leq_{i}$ denotes the immersion ordering?

\section{JUMP NUMBER}

Instance: A poset $P=(P, \leq)$.

Parameter: A positive integer $k$.

Question: Is the jump number of $P \leq k$ ?

The general version of this problem is NP-complete (See Pulleybank [103]). By El-Zahar and Schmerl [67], there is an $O\left(n^{k+1}\right)$ algorithm.

\section{PLANAR $t$-NORMALIZED WEIGHTED SATISFIABILITY}

Instance: A planar $t$-normalized formula $X$.

Parameter: A positive integer $k$.

Question: Does $X$ have a satisfying assignment of weight $k$ ?

This question is of some interest for $t=1$ since it might be a candidate for an intractable problem that is not $W[1]$ hard.

\section{PLANAR MULTIWAY CUT}

Instance: A weighted planar graph $G=(V, E)$ with terminals $\left\{x_{1}, \ldots, x_{k}\right\}$.

Parameter: $k$

Question: Is there a set of edges of total weight $\leq k^{\prime}$ whose removal disconnects each terminal from all the others?

The general version of this problem is $N P$-complete (Dalhaus et al. [50]). Best known complexity is $O\left(\left(4^{k}\right)^{k} n^{2 k-1} \log n\right)$ by [50] where it is asked if the problem is $F P T$. 


\section{POLYMATROID RECOGNITION}

Instance: A $k$-polymatroid $M$.

Parameter: A positive integer $k$.

Question: Is $M$ hypergraphic?

See Vertigan and Whittle [116].

\section{RESTRICTED VALENCE ISOMORPHISM}

Instance: Two graphs $G=(V, E)$ and $H=\left(V^{\prime}, E^{\prime}\right)$.

Parameter: A positive integer $k$.

Question: Are $G$ and $H$ isomorphic graphs such that the valences of the vertices of both $G$ and $H$ are bounded by $k$ ?

Luks [97] has shown that there is an $O\left(n^{f(k)}\right)$ algorithm to decide the parameterized version. The question is whether the problem is $F P T$. If this problem is $W[1]$-hard then GRAPH ISOMORPHISM is not in $P$ unless the $W$-Hierarchy collapses. The reader should note that this may give an ingress into the GRAPH ISOMORPHISM problem, in the sense one might be able to demonstrate intractability (i.e., assuming that $W[1] \neq F P T$ ) of the unparameterized version by considering some parameterized version instead. The point is that we have already seen many instances where the "complexity" of the unparameterized version is quite different than the parameterized one.

\section{SHORT CHEAP TOUR}

Instance: A graph $G=(V, E)$, an edge weighting $w: E \rightarrow \mathbf{Z}$.

Parameter: A positive integer $k$.

Question: Is there a tour through at least $k$ nodes of $G$ of cost at most $S$ ?

The general version of this problem is $N P$-complete by a reduction from HAMILTON CIRCUIT (see Garey and Johnson [81, ND22]). Known to be hard for $W$ [1] if we ask that the tour cost exactly $S$ (Downey and Fellows [56]).

\section{SHORT GENERALIZED HEX}

Instance: A graph $G=(V, E)$ with two distinguished vertices $v_{1}$ and $v_{2}$.

Parameter: A positive integer $k$.

Question: Does player one have a winning strategy of at most $k$ moves in the following game? Player one plays with white pebbles and player two with black ones. Pebbles are placed on nondistinguished vertices alternately by player one then player two. Player one wins if he can construct a path of white vertices from $v_{1}$ to $v_{2}$.

The general version of this problem is $P S P A C E$-complete by a reduction from $\mathrm{QBF}$ (see Garey and Johnson [81, GP1]). Candidate for $A W[*]$-completeness (Abrahamson, Downey, and Fellows [2]).

\section{SMALL MINIMUM DEGREE 4 SUBGRAPH}

Instance: A graph $G=(V, E)$.

Parameter: A positive integer $k$.

Question: Is there a subgraph of $G$ of minimum degree at least 4 and of cardinality at most $k$ ?

\section{TOPOLOGICAL CONTAINMENT}

Instance: A graph $G=(V, E)$.

Parameter: A graph $H$.

Question: Is $H$ topologically contained in $G$ ?

This can be solved in $O\left(n^{O\left(\left|E^{\prime}\right|\right)}\right)$ time by brute force together with the $k$-DISJOINT PATHS algorithm of Robertson and Seymour [106]. 


\section{WEIGHTED MONOTONE PLANAR BOOLEAN CIRCUIT SATISFIABILITY}

Instance: A monotone planar decision circuit $C$.

Parameter: A positive integer $k$.

Question: Does $C$ have a satisfying assignment of weight $k$ ?

Candidate for $W[S A T]$-completeness (Abrahamson, Downey, and Fellows [2]).

Acknowledgments. Thanks to Karl Abrahamson for useful early discussions about this work, and for suggestions on improving the exposition. Special thanks to Mike Hallett and $\mathrm{H}$. Todd Wareham, who prepared the final version of the appendix.

\section{REFERENCES}

[1] K. A. Abrahamson, R. G. Downey, and M. R. Fellows, Fixed-Parameter Intractability II, in STACS 93, Lecture Notes in Computer Science, Springer-Verlag, Berlin, 1993, pp. 374-385.

$[2]-$ Fixed-parameter tractability and completeness IV: On completeness for $W[P]$ and PSPACE analogues, Ann. Pure Appl. Logic, to appear.

[3] K. A. Abrahamson, J. Ellis, M. R. Fellows, and M. Mata, On the complexity of fixed-parameter problems, in 30th Annual Symposium on Foundations of Computer Science, IEEE Press, Piscataway, NJ, 1989, pp. 210-215.

[4] K. A. Abrahamson and M. R. Fellows, Cutset regularity beats well-quasi-ordering for bounded treewidth, Tech. Report, Computer Science Dept., Univ. of Idaho, 1989.

[5] —- Finite automata, bounded treewidth, and well-quasiordering, in Graph Structure Theory, N. Robertson and P. Seymour, eds., Contemporary Mathematics Vol. 147, American Mathematical Society, Providence, RI, 1993, pp. 539-564.

[6] R. Anderson And E. MAYR, A P-complete problem and approximations to it, Stanford University, Tech. Report STAN-CS-84-1014, 1984.

[7] S. ARNBORG, Efficient algorithms for combinatorial problems on graphs with bounded decomposability - a survey, BIT, 25 (1985), pp. 2-23.

[8] S. ARnborg, D. G. CorneIl, AND A. Proskurowski, Complexity of finding embeddings in a $k$-tree, SIAM J. Alg. Discrete Meths., 8, (1987), pp. 277-284.

[9] S. Arnborg, B. Courcelle, A. Proskurowski, and D. Seese, An Algebraic Theory of Graph Reduction, Tech. Report 90-02, Laboratoire Bordelais de Recherche Informatique, Univ. Bordeaux I, January 1990.

[10] S. Arnborg, J. Lagergren, ANd D. Seese, Problems easy for tree-decomposible graphs (extended abstract), in Proc. 15th Coll. Automata, Languages and Programming, T. Lepisto and A. Salomaa, eds., Lecture Notes in Computer Science, Vol. 317, Springer-Verlag, Berlin, 1988, pp. 38-51.

[11] S. Ausiello, A. D'ATRI, AND M. PROTASI, Structure preserving reductions among convex optimization problems, J. Comput. System Sci., 21 (1980), pp. 136-153.

[12] J. BalcazaAr, J. Diaz, AND J. Gabarro, Structural Complexity, Vols. 1 and 2, Springer-Verlag, Berlin, 1987 , 1989.

[13] M. W. BERN, E. L. LAWLER, AND A. L. WONG, Linear time computation of optimal subgraphs of decomposable graphs, J. Algorithms, 8 (1987), pp. 216-235.

[14] D. Bienstock AND M. A. LANGSTON, Algorithmic implications of the graph minor theorem, in Handbook of Operations Research and Management Science: Volume on Networks and Distribution, to appear.

[15] D. BIENSTOCK AND C. MONMA, On the complexity of covering vertices by faces in planar graphs, SIAM J. Comput., 17 (1988), pp. 53-76.

[16] H. L. BoDlaENDER, On linear time minor tests and depth-first search, in Proc. 1st Workshop on Algorithms and Data Structures, F. Dehne et al., eds., Lecture Notes in Computer Science, Vol. 382, Springer-Verlag, Berlin, 1987, pp. 577-590.

[17] - Classes of Graphs with Bounded Tree-Width, Tech. Report RUU-CS-86-22, Dept. of Computer Science, Univ. of Utrecht, Utrecht, The Netherlands, 1986.

[18] - Dynamic programming on graphs with bounded tree-width, in Proc. 15th Int. Coll. Automata, Languages and Programming, T. Lepistö and A. Salomaa, eds., Lecture Notes in Computer Science, Vol. 317, Springer-Verlag, Berlin, 1988, pp. 103-118.

[19] - Some classes of graphs with bounded treewidth, Bull. EATCS, 36 (1988), pp. 116-126.

[20] - On Disjoint Cycles, Tech. Report RUU-CS-90-29, Dept. of Computer Science, Univ. of Utrecht, Utrecht, The Netherlands, August 1990. 
[21] H. L. BoDLAENDER, private communication, 1994.

[22] — A Tourist's Guide Through Treewidth, Tech. Report RUU-CS-92-12, Computer Science Department, Univ. of Utrecht, Utrecht, The Netherlands, March 1992.

[23] - A linear time algorithm for finding tree-decompositions of small treewidth, in Proceedings of the 25th ACM Symposium on Theory of Computing, ACM Press, 1993, pp. 226-234.

[24] H. L. Bodlaender, R. G. Downey, M. R. Fellows, M. T. Hallett, and H. T. Wareham, Parameterized complexity analysis in computational biology, in The IEEE Workshop on Shape and Pattern Matching in Computational Biology, held in conjunction with 1994 IEEE Conference on Computer Vision and Pattern Recognition, June 1994.

[25] H. L. Bodlaender, R. G. Downey, M. R. Fellows, and H. T. Wareham, The parameterized complexity of sequence alignment and consensus (extended abstract), in Proceedings of the Fifth Conference on Combinatorial Pattern Matching (CPM '94), Springer-Verlag, Lecture Notes in Computer Science 807, 1994, pp. 15-30.

[26] - The parameterized complexity of sequence alignment and consensus, Theoret. Comput. Sci, to appear.

[27] H. L. Bodlaender And J. Engelfriet, Domino Treewidth, Tech. Report UU-CS-1994-11, Department of Computer Science, University of Utrecht, Utrecht, The Netherlands, 1994.

[28] H. L. BodlaEnder, M. R. Fellows, AND M. T. HalletT, Beyond NP-completeness for problems of bounded width: Hardness for the W-hierarchy (extended abstract), STOC 26, 1994, pp. 449-458.

[29] H. L. Bodlaender, M. R. Fellows, And T. Warnow, Two Strikes Against Perfect Phylogeny, Tech. Report RUU-CS-92-08, Department of Computer Science, University of Utrecht, Utrecht, The Netherlands, 1992.

[30] H. L. Bodlaender AND D. KRatsch, private communication, 1994.

[31] R. B. BORIE, R. G. PARKER AND C. A. TOVEY, Automatic generation of linear-time algorithms from predicate calculus descriptions of problems on recursively constructed graph families, Algorithmica, 7 (1992), pp. 555-582.

[32] G. Brightwell AND D. Winkler, Counting linear extensions, Order, 8 (1991), pp. 225-242.

[33] - Counting linear extensions is \#P-complete, Proc. 23rd STOC, IEEE Press, Piscataway, NJ, 1991, pp. $175-181$.

[34] S. Buss, private communication, 1989.

[35] J. F. Buss AND J. GoldSMITH, Nondeterminism within P, SIAM J. Comput., 22 (1993), pp. 560-572.

[36] L. CAI, Fixed parameter tractability and approximation problems, Project Report, Computer Science Department, Texas A\&M University, June 1992.

[37] L. CAI AND J. CHEN, On the amount of nondeterminism and the power of verifying, Lecture Notes in Computer Science, Vol. 711 (MFCS'93), Springer-Verlag, Berlin, 1993, pp. 311-320.

[38] - On Input Read Modes of Alternating Turing Machines, Tech. Report 93-046, Department of Computer Science, Texas A\&M University, College Station, TX, 1993.

[39] - Fixed-parameter tractability and approximability of NP-hard optimization problems, Proceedings of the 2nd Israel Symposium on Theory of Computing and Systems, 1993, pp. 118-126.

[40] L. CAI, J. Chen, R. G. Downey, AND M. R. Fellows, The parameterized complexity of short computation and factorization, Archive Math. Logic, to appear.

[41] - Advice classes of parameterized tractability, Annals Pure and Applied Logic, to appear.

[42] - On the structure of parameterized problems in NP, in STACS '94, Lecture Notes in Computer Science, Springer-Verlag, Berlin, 1994, pp. 509-520.

[43] K. CATtEll, M. DinNeEn, AND M. R. Fellows, A simple linear-time algorithm for finding path-decompositions of small width, to appear.

[44] M. CeSATI, private communication, 1994.

[45] P. ChOlAK AND R. G. Downey, Undecidability and definability for parameterized polynomial time mreducibilities, in Logical Methods, J. Crossley, J. Remmel, R. Shore and M. Sweedler eds., Birkhauser, Boston, 1994, pp. 194-221.

[46] C. CHegireddy AND H. HAMACHER, Algorithms for finding the $k$ best perfect matchings, J. Combin. Theory Ser. B., (1987), pp. 155-165.

[47] A. Condon, The complexity of the max word problem and the power of one way interactive proof systems, in STACS '91, Lecture Notes in Computer Science, Springer-Verlag, 1991.

[48] S. CooK, The complexity of theorem proving procedures, Proceedings 3rd STOC, ACM Press, 1971, pp. 151158.

[49] B. COURCELLE, Graph rewriting: An algebraic and logic approach, in Handbook of Theoretical Computer Science, Vol. B, J. van Leeuwen, ed., North-Holland, Amsterdam, 1990.

[50] E. Dahlhaus, D. Johnson, C. PAPadimitriou, P. Seymour, AND M. YannaKakis, The complexity of multiway cuts, in STOC '92, ACM Press, 1992, pp. 241-251. 
[51] M. Davis, Unsolvable problems, in The Handbook of Mathematical Logic, J. Barwise, ed., Elsevier, New York, 1977.

[52] W. DAY AND D. SANKofF, Computational complexity of inferring phylogenies by compatibility, Systematic Zoology, 352 (1986), pp. 224-229.

[53] J. Díaz AND J. TORÁn, Classes of bounded nondeterminism, Math. Systems Theory, 23 (1990), pp. 21-32.

[54] R. G. Downey, P. Evans, AND M. R. Fellows, Parameterized learning complexity, in Proceedings of the 6th Annual Conference on Learning Theory, COLT'93, ACM Press, New York, 1993, pp. 51-57.

[55] R. G. Downey AND M. R. Fellows, Fixed-parameter tractability and completeness, Congr. Numer., 87 (1992), pp. 161-187.

[56] - Fixed-parameter tractability and completeness II: On completeness for W [1], Theoret. Comput. Sci., to appear.

[57] - Fixed parameter intractability (extended abstract), in Proc. Structure in Complexity Theory, IEEE Press, Piscataway, NJ, 1992, pp. 36-50.

[58] - Fixed-parameter tractability and completeness III: some structural aspects of the W-hierarchy, in Complexity Theory, K. Ambos-Spies, S. Homer, and U. Schoning, eds., Cambridge University Press, 1993, pp. 166-191.

[59] — Parameterized computational feasibility, in Proceedings of Feasible Mathematics II, P. Clote and J. B. Remmel, eds., Birkhauser, Boston, 1995, pp. 219-244.

[60] - Fixed-parameter tractability and intractability-a survey, in preparation.

[61] - Parameterized Complexity, monograph in preparation.

[62] R. G. Downey, M. R. Fellows, B. Kapron, M. T. Hallett, and H. T. Wareham, Parameterized complexity of some problems in logic and linguistics (extended abstract), in the 2nd Workshop on Structural Complexity and Recursion-Theoretic Methods in Logic Programming, 1993. Also in Logic at St. Petersberg, A. Nerode and Y. Matiyasevich, eds., Lecture Notes in Computer Science, Vol. 813, 1994, pp. 89-101.

[63] - Parameterized complexity of some problems in logic and linguistics, in preparation.

[64] R. G. Downey, M. R. Fellows AND K. ReGan, Parameterized circuit complexity and the W-heirarchy, to appear.

[65] S. DREYfuS AND R. WAGNER, The Steiner problem in graphs, Networks, 1 (1971), pp. 195-207.

[66] E. DuBROVA, private communication, 1994.

[67] M. El-ZAHAR AND J. SCHMERL, On the size of jump critical ordered sets, Order, 1 (1984), pp. 3-5.

[68] P. ERdös AND L. PósA, On independent circuits contained in a graph, Canad. J. Math., (1965), pp. 347-352.

[69] M. R. FELLows, The Robertson-Seymour theorems: A survey of applications, in Contemporary Mathematics, Vol. 89, American Mathematics Society, Providence, RI, 1989, pp. 1-18.

[70] - private communication, 1994.

[71] M. R. Fellows, M. T. Hallett, and H. T. Wareham, DNA physical mapping: Three ways difficult, in Proceedings: ESA'93 - European Symposium on Algorithms, T. Lengauer ed., Lecture Notes in Computer Science, Vol. 726, Springer-Verlag, Berlin, 1993, pp. 157-168.

[72] M. R. FEllowS AND N. KoBlitZ, Self-witnessing polynomial-time complexity and prime factorization, in Proc. 7th Structure in Complexity, IEEE Press, Piscataway, NJ, 1992, pp. 107-111.

[73] — Fixed-Parameter Complexity and Cryptography, Tech. Report DCS-207-IR, Department of Computer Science, University of Victoria, Victoria, BC, 1992.

[74] M. R. Fellows AND M. A. LANGSton, Nonconstructive tools for proving polynomial time decidability, J. Assoc. Comput. Mach., 35 (1988), pp. 727-739.

[75] - Layout permutation problems and well-partially ordered sets, in Proc. 5th MIT Conf. on Advanced Research in VLSI, MIT Press, 1988, pp. 315-327.

[76] - On search, decision and the efficiency of polynomial-time algorithms, in Proc. Symp. on Theory of Computing (STOC), 1989, ACM Press, pp. 501-512.

[77] - An analogue of the Myhill-Nerode theorem and its use in computing finite basis characterizations, in Proc. Symp. Foundations of Comp. Sci. (FOCS), IEEE Press, 1989, pp. 520-525.

[78] - Constructivity issues in graph algorithms, in Conf. on Constructivity Issues in Computer Science, San Antonio, TX, 1991, Lecture Notes in Computer Science, Springer-Verlag, 1991.

[79] - On well-partial-ordering theory and its applications to combinatorial problems in VLSI design, SIAM J. Discrete Math., 5 (1992), pp. 117-126.

[80] - Nonconstructive advances in polynomial-time complexity, Inform. Process. Lett., 28 (1987/88), pp. 157-162.

[81] M. R. GAREY AND D. S. JoHnSON, Computers and Intractability: A Guide to the Theory of NP-completeness, Freeman, San Francisco, 1979.

[82] W. GASARCh, M. KRENTEL, AND K. RAPPOPORT, OptP as the normal behavior of NP-complete problems, Math. Systems Theory, to appear. 
[83] J. G. GESKE, On the Structure of Intractable Sets, Ph.D. thesis, Iowa State University, Ames, IA, 1987.

[84] P. Goldberg, M. Golumbic, H. Kaplan, and R. ShamiR, Three strikes against physical mapping of DNA (extended abstract), manuscript, 1994.

[85] M. Golumbic, H. Kaplan, And R. Shamir, On the Complexity of DNA Physical Mapping, Tech. Report 271/93, Tel Aviv University, Tel Aviv, Israel, 1993.

[86] Y. GuRevich And S. Shelah, Nearly linear time, in Logic at Botik '89, A. R. Meyer and M. A. Taitslin, eds., Lecture Notes in Computer Science, Vol. 363, Springer-Verlag, Berlin, 1989, pp. 108-118.

[87] M. T. HALLETT, personal communication, 1994.

[88] J. HopCroft AND J. UllmanN, Introduction to Automata Theory, Addison-Wesley, Reading, MA, 1979.

[89] A. ITAI, M. RoDEH, AND S. TANIMOTO, Some matching problems in bipartite graphs, J. Assoc. Comput. Mach., 25 (1978), pp. 517-525.

[90] H. Kaplan and R. Shamir, Pathwidth, Bandwidth and Completion Problems to Proper Interval Graphs with Small Cliques, Tech. Report 285/93, Tel Aviv University, Tel Aviv, Israel, 1993.

[91] H. KAPLAN, R. SHAMIR, AND R. E. TARJAN, Tractability of parameterized completion problems on chordal and interval graphs: minimum fill-in and DNA physical mapping (extended abstract), Proc. 35th Symposium on Foundations of Computer Science (FOCS), IEEE Press, November 1994, pp. 780-791.

[92] R. KARP, Reducibility among combinatorial problems, in Complexity of Computer Computations, R. Miller and J. Thatcher, eds., Plenum Press, New York, 1973, pp. 45-68.

[93] C. KINTALA AND P. FISCHER, Refining nondeterminism in relativised polynomial time bounded computations, SIAM J. Comput., 9 (1980), pp. 46-53.

[94] R. LADNER, On the structure of polynomial time reducibility, J. Assoc. Comput. Mach., 22 (1975), pp. 155-171.

[95] J. LAGERGREN, Algorithms and minimal forbiddent minors for tree decomposible graphs, Ph.D. Dissertation, Dept. of Numerical Analysis and Computing Sciences, Royal Institute of Technology, Stockholm, Sweden, March 1991.

[96] H. R. LEWIS, Complexity results for classes of quantificational formulas, J. Comput. Systems Sci., 21 (1980), pp. 317-353.

[97] E. LuKs, Isomorphism of graphs of bounded valence can be tested in polynomial time, J. Comput. Systems Sci., 25 (1982), pp. 42-65.

[98] A. NAIK, K. REGAN, AND D. SIVAKUMAR, Quasilinear time complexity theory, in STACS '94, Lecture Notes in Computer Science, Springer-Verlag, Berlin, 1994, pp. 97-108.

[99] J. NeSETRIL AND S. PoljaK, On the complexity of the subgraph problem, Comm. Math. Univ. Carol., 26 (1985), pp. 415-419.

[100] C. PAPAdimitriou AND M. YANNAKAKIS, On limited nondeterminism and the complexity of the V-C dimension, Eighth Annual Conference on Structure in Complexity Theory, IEEE Press, 1993 pp. 12-18.

[101] A. PAZ AND S. MORAN, Nondeterministic polynomial optimization problems and their approximations, Theoret. Comput. Sci., 15 (1981), pp. 251-277.

[102] J. PLEHN AND B. VoIGT, Finding minimally weighted subgraphs, in Proc. 16th International Workshop WG90, Graph-Theoretic Concepts in Computer Science, Lecture Notes in Computer Science 484, SpringerVerlag, (1991), pp. 18-29.

[103] B. Pulleybank, On minimizing setups in precedence constrained scheduling, Discrete Appl. Math., 11 (1985).

[104] K. Regan, Finitary substructure languages, in Proc. 4th Structure in Complexity Conf., IEEE Press, Piscataway, NJ, 1989, pp. 87-96.

[105] E. RISTAD, Complexity of Simplified Segmental Phonology. Tech. Report CS-TR-388-92, Dept. of Computer Science, Princeton University, Princeton, NJ, revised May 1993.

[106] N. RoberTSON AND P. D. SeYMour, Disjoint paths - a survey, SIAM J. Discrete Math., 6(1985), pp. 300-305.

[107] - Graph minors XIII: The disjoint paths problem, to appear.

[108] - Graph minors XV: Wagner's conjecture, to appear.

[109] H. U. SIMON, Continuous reductions among combinatorial optimization problems, Acta Informatica, 26 (1989), pp. 771-785.

[110] M. SIPSER, Borel sets and circuit complexity, in Proceedings 15th STOC, ACM Press, 1983, pp. 61-69.

[111] W. SLOUGH AND K. WinkLMANN, On limitations of transformations between combinatorial problems, Journal Comput. System Sci., to appear.

[112] E. SPILRAJN, Sur l'extension de l'ordre partiel, Fund. Math., 16 (1930), pp. 386-389.

[113] L. VAliant, The complexity of computing the permanent, Theoret. Comput. Sci., 8 (1979), pp. 189-201.

[114] L. Valiant and V. V. Vazirani, NP is as easy as detecting unique solutions, Theoret. Comput. Sci., 47 (1986), pp. 85-93.

[115] L. VAN LeEuwen, Handbook of Theoretical Computer Science, Vol. A, North Holland, Amsterdam, 1990. 
[116] D. Vertigan AND G. WhItTLE, Recognising polymatroids associated with hypergraphs, Combin., Probab. Comput., to appear.

[117] H. T. WAREHAM, personal communication, 1994.

[118] T. WiMER, S. HEDETNIEMI, AND R. LASKAR, A methodology for constructing linear time algorithms, Congr. Numer., 50 (1985), pp. 43-60.

[119] M. YannaKaKIS, Computing the minimum fill-in is NP complete, SIAM J. Alg. Discrete Meth., 2 (1981), pp. 77-79. 